\title{
Dark Matter in a twisted bottle
}

\author{
Alexandre Arbey, ${ }^{a, b, c}$ Giacomo Cacciapaglia, ${ }^{d}$ Aldo Deandrea ${ }^{d}$ and Bogna Kubik ${ }^{d}$ \\ ${ }^{a}$ Centre de Recherche Astrophysique de Lyon, Observatoire de Lyon, Université Lyon 1, \\ CNRS, UMR 5574, \\ 9 avenue Charles André, Saint-Genis Laval cedex, F-69561, France \\ ${ }^{b}$ Ecole Normale Supérieure de Lyon, \\ Lyon, France \\ ${ }^{c}$ Theory Division, Physics Department, CERN, \\ CH-1211 Geneva 23, Switzerland \\ 'Université Lyon 1, CNRS/IN2P3, UMR5822 IPNL, \\ Lyon, F-69622 Villeurbanne Cedex, France \\ E-mail: alexandre.arbey@ens-lyon.fr, g.cacciapaglia@ipnl.in2p3.fr, \\ deandrea@ipnl.in2p3.fr, bkubik@ipnl.in2p3.fr
}

ABSTRACT: The real projective plane is a compact, non-orientable orbifold of Euler characteristic 1 without boundaries, which can be described as a twisted Klein bottle. We shortly review the motivations for choosing such a geometry among all possible two-dimensional orbifolds, while the main part of the study will be devoted to dark matter study and limits in Universal Extra Dimensional (UED) models based on this peculiar geometry. In the following we consider such a UED construction based on the direct product of the real projective plane with the standard four-dimensional Minkowski space-time and discuss its relevance as a model of a weakly interacting Dark Matter candidate.

One important difference with other typical UED models is the origin of the symmetry leading to the stability of the dark matter particle. This symmetry in our case is a remnant of the six-dimensional Minkowski space-time symmetry partially broken by the compactification. Another important difference is the very small mass splitting between the particles of a given Kaluza-Klein tier, which gives a very important role to co-annihilation effects. Finally the role of higher Kaluza-Klein tiers is also important and is discussed together with a detailed numerical description of the influence of the resonances.

KEYwords: Phenomenology of Field Theories in Higher Dimensions 


\section{Contents}

1 Introduction 2

2 Dark Matter relic density 3

3 UED on a twisted bottle $\quad 5$

$\begin{array}{lll}3.1 & \text { Field content } & 7\end{array}$

4 Particle spectrum and the Dark Matter candidate $\quad 8$

4.1 Decoupling limit $R_{4} \gg R_{5} \quad 9$

4.2 Degenerate radii $R_{4}=R_{5} \quad 9$

4.3 Full numerical spectra and bound on parameters of the model 10

5 Analytical results $\quad \mathbf{1 5}$

5.1 LKP annihilations - analytic expressions for the cross sections 16

$\begin{array}{lll}5.1 .1 & A^{(1)} A^{(1)} \rightarrow Z Z & 16\end{array}$

$\begin{array}{lll}5.1 .2 & A^{(1)} A^{(1)} \rightarrow W^{+} W^{-} & 17\end{array}$

$\begin{array}{lll}5.1 .3 \quad A^{(1)} A^{(1)} \rightarrow h h & 18\end{array}$

$\begin{array}{lll}5.1 .4 & A^{(1)} A^{(1)} \rightarrow f \bar{f} & 18\end{array}$

$\begin{array}{lll}5.2 & \text { Influence of KK mass degeneracy and Higgs contribution } & 19\end{array}$

6 Relic abundance - numerical results $\quad 23$

$\begin{array}{lll}6.1 & \text { L1 scenario } 24\end{array}$

6.1.1 Influence of co-annihilations 24

6.1.2 Influence of the resonances of (2) modes and loop induced couplings 26

$\begin{array}{lll}6.2 \text { L2 scenario } & 29\end{array}$

6.3 Comparison of $m_{K K}$ bounds in L1 and L2 scenarios 31

7 Cut-off dependence of the relic abundance $\quad 32$

8 Localised Higgs mass dependence $\quad 34$

$\begin{array}{lll}9 & \text { Direct detection bounds } & 37\end{array}$

$\begin{array}{ll}10 \text { Conclusion } & 38\end{array}$ 


\section{Introduction}

The motivations for building a specific model based on a compactified Extra Dimension can be of different origins, ranging from phenomenological ones to more formal ones related for example to string theory. For instance, the issue of the radiative stability of the Higgs boson mass or the mass hierarchy in the fermion and gravity sectors can be addressed in novel ways compared to 4 dimensional physics. In this paper we focus on the issue of Dark Matter in Universal Extra Dimension (UED) type of models. In the following we shall consider an effective theory defined on a $d$ dimensional manifold which is the direct product of the standard four-dimensional Minkowski space-time $\mathcal{M}^{4}$ and a $(d-4)$-dimensional orbifold defined as the quotient space of $\mathbb{R}^{(d-4)}$ modulo a discrete symmetry group $\Gamma$. This general framework can be constrained by few important theoretical requirements which will select, in our case, a unique geometry.

One of the most attractive explanations to the presence of Dark Matter (DM) in the universe is the existence of a weakly interacting particle (WIMP), present in many extensions of the Standard Model. A main requirement for a particle theory model is therefore the possibility to obtain, in a natural way, a viable Dark Matter candidate, in the form of a neutral, stable and weakly interacting particle. From a theoretical point of view, its stability should be obtained as the result of a symmetry conservation law. In this respect many models impose an ad hoc parity, which may or may not have other independent justifications. In extra dimensions, residual symmetries of the compact space can play the role of the parity that stabilises a Dark Matter candidate [1], however such symmetry usually requires ad hoc constraints on the effective Lagrangian of the model. The scenario we discuss here, based on the real projective plane orbifold, has a special status as the stability of the dark matter candidate is not imposed, but is the result of an exact residual space-time symmetry after compactification.

The presence of exact residual symmetries is related to fixed points or lines. Fixed points, which are points in the extra space which are left invariant by all the symmetries of the orbifold projection, in general break the $d$-dimensional Lorentz invariance to the 4 dimensional one, therefore no extra symmetry would survive in general and the predictivity of the model is considerably reduced. Indeed the divergences appearing in loop corrections require counter-terms localised on these fixed points. Another important theoretical and phenomenological requirement is the presence of 4-dimensional chiral fermions as zero modes in the low energy spectrum of the effective theory. The requirements of the absence of fixed points and of the presence of chiral zero modes completely eliminate the possibility of working with only one compact extra dimension, as the only 1-dimensional orbifold without fixed points is the circle $S^{1}$. However no chiral fermions can be defined on the circle without taking a quotient introducing fixed points. In [2] it was shown that the unique orbifold without fixed points, among the 17 orbifolds which can be defined on a 2-dimensional euclidean plane, is the real projective plane $\left(\mathrm{RP}^{2}\right)$. One may consider higher dimensional orbifolds, but naive dimensional analysis shows quite easily that increasing the space-time dimensionality of the effective theory brings automatically an increase in the dimension of the fields and operators which reduces drastically the predictivity of an 
effective theory based on the quantum field theory paradigm. We shall therefore limit our study to this unique 2-dimensional orbifold, the real projective plane. Its fundamental domain is a rectangle with opposite sides identified after being twisted, like a "double" Möbius strip. If we were to twist only along one direction, and join the other two sides plainly, we would obtain a Klein bottle: therefore, the real projective plane can also be thought as a twisted Klein bottle.

The paper is organised as follows: after summarising in section 2 the basic formulas for the DM relic density calculation, in section 3 we briefly present the real projective plane and the corresponding UED model. In section 4 we describe in detail the peculiar spectrum of the $\mathrm{RP}^{2}$ model, its dark matter particle candidate (LKP) and the main formulas for its pair annihilation. In the following sections 5 and 6 we study the relic abundance using respectively analytic and numerical calculations. These two sections complete nicely each other for the understanding of the relic abundance behaviour of different contributions: annihilation versus co-annihilation and different particles contributions for DM observables. The effect of the cut-off of the effective theory is discussed in section 7, while that of the localised Higgs mass in section 8. In section 9 we consider the present and future direct detection bounds. Section 10 contains our conclusions.

\section{Dark Matter relic density}

In order to compute the dark matter relic density, we assume the cosmological standard model, which is based on a Friedmann-Lemaitre Universe filled with radiation, baryonic matter and cold dark matter, approximately flat and incorporating a cosmological constant accelerating its expansion. Before recombination, the Universe expansion is dominated by a radiation density, and therefore the expansion rate $H$ of the Universe is determined by the Friedmann equation

$$
H^{2}=\frac{8 \pi G}{3} \rho_{\mathrm{rad}}
$$

where

$$
\rho_{\mathrm{rad}}(T)=g_{\mathrm{eff}}(T) \frac{\pi^{2}}{30} T^{4}
$$

is the radiation density and $g_{\text {eff }}$ is the effective number of degrees of freedom of radiation. The computation of the relic density is based on the solution of the Boltzmann evolution equation $[3,4]$

$$
d n / d t=-3 H n-\left\langle\sigma_{\mathrm{eff}} v\right\rangle\left(n^{2}-n_{\mathrm{eq}}^{2}\right),
$$

where $n$ is the number density of all KK particles, $n_{\text {eq }}$ their equilibrium density, and $\left\langle\sigma_{\text {eff }} v\right\rangle$ is the thermal average of the annihilation rate of the KK particles to the Standard Model particles. The thermal average of the effective cross section is given by

$$
\left\langle\sigma_{\mathrm{eff}} v\right\rangle=\frac{\int_{0}^{\infty} d p_{\mathrm{eff}} p_{\mathrm{eff}}^{2} W_{\mathrm{eff}}(\sqrt{s}) K_{1}\left(\frac{\sqrt{s}}{T}\right)}{m_{\mathrm{LKP}}^{4} T\left[\sum_{i} \frac{g_{i}}{g_{\mathrm{LKP}}} \frac{m_{i}^{2}}{m_{1}^{2}} K_{2}\left(\frac{m_{i}}{T}\right)\right]^{2}},
$$


where $K_{1}$ and $K_{2}$ are the modified Bessel functions of the second kind of order 1 and 2 respectively, and

$$
\frac{d W_{\text {eff }}}{d \cos \theta}=\sum_{i j k l} \frac{p_{i j} p_{k l}}{8 \pi g_{\mathrm{LKP}}^{2} p_{\text {eff }} S_{k l} \sqrt{s}} \sum_{\text {helicities }}\left|\sum_{\text {diagrams }} \mathcal{M}(\tilde{i} \tilde{j} \rightarrow k l)\right|^{2},
$$

where $\mathcal{M}(\tilde{i} \tilde{j} \rightarrow k l)$ is the transition amplitude of the (co-)annihilation of KK particles $\tilde{i}$ and $\tilde{j}$ into SM particles $k$ and $l$,

$$
g_{\mathrm{LKP}}^{2} p_{\mathrm{eff}} W_{\mathrm{eff}} \equiv \sum_{i j} g_{i} g_{j} p_{i j} W_{i j}
$$

with

$$
p_{\text {eff }}(\sqrt{s})=\frac{1}{2} \sqrt{(\sqrt{s})^{2}-4 m_{\mathrm{LKP}}^{2}}
$$

and where $\theta$ is the angle between particles $\tilde{i}$ and $k$. By solving the Boltzmann equation, the density number of KK particles in the present Universe and consequently the relic density can be determined. The ratio of the number density to the radiation entropy density, $Y(T)=n(T) / s(T)$ can be defined, where

$$
s(T)=h_{\mathrm{eff}}(T) \frac{2 \pi^{2}}{45} T^{3} .
$$

$h_{\text {eff }}$ is the effective number of entropic degrees of freedom of radiation. Combining eqs. (2.1) and (2.3) and defining $x=m_{\mathrm{LKP}} / T$, the ratio of the LKP mass over temperature, yield

$$
\frac{d Y}{d x}=-\sqrt{\frac{\pi}{45 G}} \frac{g_{*}^{1 / 2} m_{\mathrm{LKP}}}{x^{2}}\left\langle\sigma_{\mathrm{eff}} v\right\rangle\left(Y^{2}-Y_{\mathrm{eq}}^{2}\right)
$$

with

$$
g_{*}^{1 / 2}=\frac{h_{\mathrm{eff}}}{\sqrt{g_{\mathrm{eff}}}}\left(1+\frac{T}{3 h_{\mathrm{eff}}} \frac{d h_{\mathrm{eff}}}{d T}\right) .
$$

The freeze-out temperature $T_{f}$ is the temperature at which the LKP leaves the initial thermal equilibrium when $Y\left(T_{f}\right)=(1+\delta) Y_{\mathrm{eq}}\left(T_{f}\right)$, with $\delta \simeq 1.5$. The relic density is obtained by integrating eq. (2.9) from $x=0$ to $m_{\mathrm{LKP}} / T_{0}$, where $T_{0}=2.726 \mathrm{~K}$ is the temperature of the Universe today $[3,4]$ :

$$
\Omega_{\mathrm{LKP}} h^{2}=\frac{m_{\mathrm{LKP}} s\left(T_{0}\right) Y\left(T_{0}\right) h^{2}}{\rho_{c}^{0}} \approx 2.755 \times 10^{8} \frac{m_{\mathrm{LKP}}}{1 \mathrm{GeV}} Y\left(T_{0}\right),
$$

where $\rho_{c}^{0}$ is the critical density of the Universe, such as

$$
H_{0}^{2}=\frac{8 \pi G}{3} \rho_{c}^{0}
$$

$H_{0}$ being the Hubble constant. The obtained relic density $\Omega_{\mathrm{LKP}} h^{2}$ can then be directly compared to the observed dark matter density. The numerical calculation of the relic density is performed using MicrOMEGAs v2.4.1 $[5,6]$. To constrain the relic density, 
we consider the 7-year WMAP data (WMAP7), which have provided an unprecedented measurement of the cold dark matter density [7]:

$$
\Omega_{c d m} h^{2}=0.1123 \pm 0.0035
$$

Taking into consideration $10 \%$ of theoretical uncertainty in the relic density calculation, we impose the following constraint:

$$
0.0773<\Omega h^{2}<0.1473
$$

It is important to remark however that the calculation of the relic density rely on many cosmological assumptions. In particular, different cosmological scenarios can lead to a relic density which is larger than that computed in the standard cosmological scenario. First, the LKP could be only one of several dark matter components. Then, if dark energy were the dominant component at the time of the relic freeze-out, it would result in an acceleration of the expansion of the Universe, which would lead to an earlier freeze-out and a much larger relic density [8-12]. Finally, entropy generation at the time of freeze-out, for example due to the decay of a late inflaton, can also lead to an increase - or a decrease of the relic density [13-17]. These effects are however limited by Big-Bang nucleosynthesis constraints, but using SuperIso Relic [18, 19] and AlterBBN [20], it can be verified that they can nevertheless lead to an increase of three orders of magnitudes or more of the relic density while still being compatible with BBN constraints. For this reason, the lower dark matter density bound can be considered as a weak constraint.

\section{UED on a twisted bottle}

The real projective plane $R P^{2}$ is a compact, non-orientable orbifold of Euler characteristic 1 without boundaries. It can be constructed in two ways, either starting from a sphere $S^{2}$ or from an infinite plane $\mathbb{R}^{2}$. The two constructions are not equivalent as in the first case the curvature is distributed on the surface while in the second we have a flat metric $g^{M N}=$ $\operatorname{diag}(1,-1,-1,-1,-1,-1)$ except for two conical singularities (but not fixed points) where curvature is concentrated. The Kaluza-Klein spectrum is also different: starting on the sphere $S^{2}$, the modes are labelled by angular momentum, while starting on the plane $\mathbb{R}^{2}$ the modes are labelled by quantised momentum along the two directions. In this paper we select the simplest case from the point of view of discussing fermions, so we choose the flat version of the orbifold that was described in ref. [2]. Some aspects of the spherical $\mathrm{RP}^{2}$ are discussed in ref. [21].

The "flat" real projective plane is defined as a quotient space $R P^{2}=\mathbb{R}^{2} / \Gamma_{R P^{2}}$ where $\Gamma_{R P^{2}}$ is a discrete symmetry group defined by two symmetry generators $g$ and $r$ as:

$$
\Gamma_{R P^{2}}=\left\langle r, g \mid r^{2}=\left(g^{2} r\right)^{2}=1\right\rangle .
$$



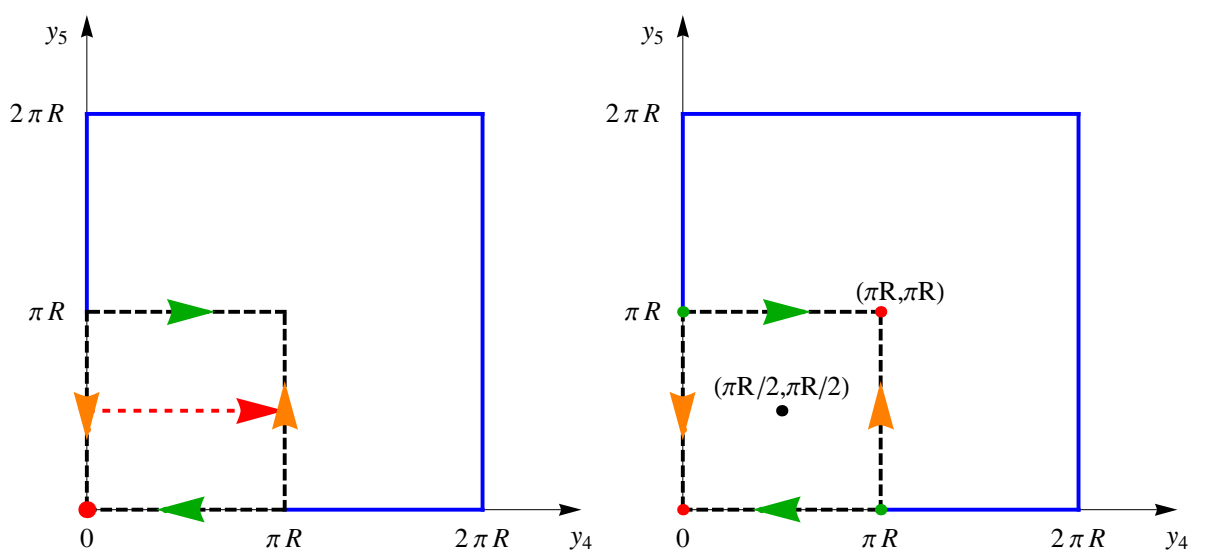

Figure 1. On the left panel: fundamental domain of the real projective plane (within black dashed lines) embedded in a torus (blue square). The red dot is $\pi$-rotation generator; the red dashed arrow represents the glide generator $g$. The green and orange arrows indicate the identification of the facing sides. On the right panel: geometrical properties of the real projective plane. The identified pairs of singular points are marked by red and green dots. The centre of the square (black dot) is the $p_{K K}$ parity centre. In this figure we set $R_{4}=R_{5}=R$.

We choose a particular representation of the generators in terms of the isometries of the plane: ${ }^{1}$

$$
g:\left\{\begin{array}{l}
x_{4} \sim g\left(x_{4}\right)=-x_{4}+\pi R_{4} \\
x_{5} \sim g\left(x_{5}\right)=x_{5}+\pi R_{5}
\end{array}, \quad r:\left\{\begin{array}{l}
x_{4} \sim r\left(x_{4}\right)=-x_{4} \\
x_{5} \sim r\left(x_{5}\right)=-x_{5}
\end{array}\right.\right.
$$

so that $g$ generates the glide (mirror reflection + translation) and $r$ corresponds to the rotation of $\pi$ degrees around the origin. The fundamental domain of our twisted bottle can be visualised as a rectangle of sides of length $\pi R_{4}$ and $\pi R_{5}$, with opposite sides identified with a twist. The orbifold obtained in this way has no fixed points and no fixed lines as can be seen in figure 1. In fact, the glide makes sure that no point can be identified with itself. The corners of the rectangle, which are fixed points of the rotation, are identified by the glide: $(0,0) \sim\left(\pi R_{4}, \pi R_{5}\right)$ and $\left(0, \pi R_{5}\right) \sim\left(\pi R_{4}, 0\right)$. These two physically nonequivalent points form two conical singularities with deficit angle $\pi$, which concentrate the curvature of the real projective plane but keeping the metric finite on these points.

The two symmetries $g$ and $r$ generate two translations $t_{4} \equiv(g * r)^{2}$ and $t_{5} \equiv g^{2}$ along the two directions $x_{4}$ and $x_{5}$ respectively under which the space is periodic, therefore the real projective plane can be embedded in a torus $T^{2}$. This is an important property as the torus is an orientable orbifold and fermions can therefore be defined in the same way as on the $T^{2}$ orbifold. The chirality of the 4-dimensional fermions can be properly defined on this space-time geometry thanks to the rotation projection $r$ (see ref. [2] for details).

\footnotetext{
${ }^{1}$ Note that the structure of the group is entirely defined by the relations between the generators. Their particular representation in terms of the isometries of the plane is not necessary but helps in visualisation.
} 


\begin{tabular}{|c|c|c|c|c|c|}
\hline$(k, l)$ & $p_{K K}$ & $(++)$ & $(+-)$ & $(-+)$ & $(--)$ \\
\hline$(0,0)$ & + & $\frac{1}{2 \pi}$ & & & \\
\hline$(0,2 l)$ & + & $\frac{1}{\sqrt{2} \pi} \cos 2 l x_{6}$ & & & $\frac{1}{\sqrt{2} \pi} \sin 2 l x_{6}$ \\
\hline$(0,2 l-1)$ & - & & $\frac{1}{\sqrt{2} \pi} \cos (2 l-1) x_{6}$ & $\frac{1}{\sqrt{2} \pi} \sin (2 l-1) x_{6}$ & \\
\hline$(2 k, 0)$ & + & $\frac{1}{\sqrt{2} \pi} \cos 2 k x_{5}$ & & $\frac{1}{\sqrt{2} \pi} \sin 2 k x_{5}$ & \\
\hline$(2 k-1,0)$ & - & & $\frac{1}{\sqrt{2} \pi} \cos (2 k-1) x_{5}$ & & $\frac{1}{\sqrt{2} \pi} \sin (2 k-1) x_{5}$ \\
\hline$(k, l)_{\mathrm{k}+1 \text { even }}$ & + & $\frac{1}{\pi} \cos k x_{5} \cos l x_{6}$ & $\frac{1}{\pi} \sin k x_{5} \sin l x_{6}$ & $\frac{1}{\pi} \sin k x_{5} \cos l x_{6}$ & $\frac{1}{\pi} \cos k x_{5} \sin l x_{6}$ \\
\hline$(k, l)_{\mathrm{k}+1 \text { odd }}$ & - & $\frac{1}{\pi} \sin k x_{5} \sin l x_{6}$ & $\frac{1}{\pi} \cos k x_{5} \cos l x_{6}$ & $\frac{1}{\pi} \cos k x_{5} \sin l x_{6}$ & $\frac{1}{\pi} \sin k x_{5} \cos l x_{6}$ \\
\hline
\end{tabular}

Table 1. Classification of the modes with parity assignment $\left(p_{r}, p_{g}\right)$ and $\mathrm{KK}$ number $(k, l)$ and corresponding normalised wave functions for a scalar field (here for simplicity we assume $R_{4}=$ $\left.R_{5}=1\right)$.

\subsection{Field content}

We are interested in a Universal Extra Dimensions model where all the Standard Model particles are allowed to propagate in the two extra dimensions. We consider a minimal version of the model that can be defined on $R P^{2}$, that is we introduce only the fields whose zero modes will reproduce the Standard Model content. The detailed construction of the orbifold and quantum fields decomposition is presented in ref. [2]. Here we mention the important points about the spectrum for the purpose of this paper.

Each quantum field is now a 6-dimensional field which, while projected on the orbifold, decomposes in an infinite tower of massive Kaluza-Klein (KK) modes. Each tier of modes is labelled by two integers $(k, l)$ which correspond to the discretised momenta along the extra directions. While projected on the orbifold, the fields can have in general four different parities under orbifold projection: $\left(p_{r}, p_{g}\right)=( \pm 1, \pm 1)$. The parities are chosen in such a way that the zero mode spectrum corresponds to the SM.

To each SM field there corresponds exactly one six-dimensional field and therefore one tower of massive KK resonances. The only exception are fermions: we have to introduce two six-dimensional spinors $\Psi^{6 D}=\left(\chi_{+}, \bar{\eta}_{-}, \chi_{-}, \bar{\eta}_{+}\right)^{T}$ with opposite rotation parities for each four-dimensional Dirac spinor. In this notation \pm subscripts correspond to the $6 \mathrm{D}$ chiralities while $\chi, \eta$ are the $4 \mathrm{D}$ chirality eigenstates. The rotation projection will cancel the the zero-more wave functions of left or right handed components $(\chi$ or $\eta)$ in $\Psi^{6 D}$ assuring a chiral 4D zero mode. For example, for a 4-dimensional Dirac spinor $e^{4 D}$ we should introduce a 6-dimensional spinor $e_{L}^{6 D}$ corresponding to a left handed component of $e^{4 D}$ and a $e_{R}^{6 D}$ corresponding to a right handed component of $e^{4 D}$, and as a result for a SM electron we will have two infinite towers of massive KK states which will differ by rotation parity assignment $p_{r}$.

The mass eigenstates can be labelled by their parity assignment $\left(p_{r}, p_{g}\right)$ and KK number $(k, l)$. As an illustration, the classification of the modes with normalised wave functions for a scalar field from [2] is (here for simplicity we fix $R_{4}=R_{5}=1$ ):

The fundamental space in figure 1, including boundaries and corners, is invariant under a $\pi$-rotation around the centre of the rectangle $r^{\prime}$. Under this rotation, however, the opposite chiralities will pick a different parity under rotation $r^{\prime}$ therefore it can not be a 
good KK parity. An equivalent symmetry can however be defined in terms of translation (see ref. [22] for details):

$$
p_{K K}=r^{\prime} * r:\left\{\begin{array}{l}
x_{4} \sim p_{K K}\left(x_{4}\right)=x_{4}+\pi R_{4} \\
x_{5} \sim p_{K K}\left(x_{5}\right)=x_{5}+\pi R_{5}
\end{array} .\right.
$$

This exact symmetry of the space can be translated into a parity on the KK states as all the fields in a $(k, l)$ mode will pick up the same phase $(-1)^{k+l}$, therefore modes with even $k+l$ are even and modes with odd $k+l$ are odd. This parity ensures the stability of the lightest odd states as they can never decay into a pair of lighter states which are even under KK parity. It follows that the lightest odd state belongs to either $(1,0)$ or $(0,1)$, thus it is in such tiers that we will look for a suitable Dark Matter candidate.

\section{Particle spectrum and the Dark Matter candidate}

In this section we present the detailed spectrum of the $(1,0)-(0,1)$ and $(2,0)-(0,2)$ tiers at one loop level. We see that the first KK excitation of the photon $A^{(1,0)}$ and/or $A^{(0,1)}$ is the viable Dark Matter candidate. We emphasise also an interesting property of the spectrum in our model, that all the particles in the same tier are nearly degenerated in mass even after the introduction of radiative corrections. This feature is important for the calculation of the relic abundance of the LKP, since the abundance is strongly affected by co-annihilation processes. Note that in other UED models $[24,25]$ the mass splitting are naturally larger.

At leading order, all the states in each tier are degenerate with mass determined by the two integers $(l, k)$ as

$$
m_{l, k}^{2}=\frac{l^{2}}{R_{4}^{2}}+\frac{k^{2}}{R_{5}^{2}}
$$

Splittings within the modes in each tier $(\mathrm{k}, \mathrm{l})$ can be generated by three mechanisms: the Higgs vacuum expectation value (VEV), bulk interaction loop corrections and higher order operators localised on the singular points.

At one loop order the mass of an $(n, 0)$ or $(0, n)$ state can be generically written as

$$
m_{(n, 0)}^{2}=\frac{1}{R_{4 / 5}^{2}}\left(n^{2}+m_{\mathrm{SM}}^{2} R_{4 / 5}^{2}+\delta_{\text {finite }}^{(n, 0)}\left(R_{4}, R_{5}\right)+n^{2} \delta_{\log }\right),
$$

where $m_{\mathrm{SM}}$ is the mass of the correspondent SM state, $\delta_{\text {finite }}$ is a finite contribution while $\delta_{\log }$ is a divergent contribution depending on the log of a cut-off scale $\delta_{\log } \sim \log \Lambda R_{4 / 5}$. Detailed formulas for the mass corrections for the tiers $(1,0)$ and $(0,1)$ can be found in ref. [23], while formulas for the even tiers $(2,0)$ and $(0,2)$ can be found in ref. [22]. We see that at loop level the masses depend on 3 free parameters: the two radii $R_{4}$ and $R_{5}$, and the cut-off $\Lambda R$. The only exceptions are the massive Higgs modes, which are only present in the even tier $(2,0)$ and $(0,2)$ : for the scalar field the most generic Lagrangian contains a mass term $\sim m_{\text {loc }}$ localised on the singular points therefore the $(2,0) \mathrm{KK}$ scalar mass at loop level is given by [22]:

$$
m_{H^{(n, 0)}}^{2}=\frac{1}{R_{4 / 5}^{2}}\left(n^{2}+m_{\mathrm{SM}}^{2} R_{4 / 5}^{2}+m_{\mathrm{loc}}^{2} R_{4 / 5}^{2}+\delta_{\text {finite }}^{(n, 0)}\left(R_{4}, R_{5}\right)+4 \delta_{\log }\right)
$$

where $m_{\text {loc }}$ is a free parameter of the model. 
As it can be seen in the formulas 4.2 and 4.3 the finite loop corrections $\delta_{\text {finite }}^{(n, 0)}$ depend on whether we consider the $(n, 0)$ or $(0, n)$ mode. However the mass correction is dominated by the log-divergent term, which is enhanced for heavier tiers while the $\delta_{\text {finite }}^{(n, 0)}$ dependence on $R_{4} / R_{5}$ is very mild [22], thus here for simplicity we consider that $\delta_{\text {finite }}^{(n, 0)}=\delta_{\text {finite }}^{(0, n)}$ and the correction relative to the tree level one is almost independent on the value of the radii $R_{4}$ and $R_{5}$.

Moreover we should consider a generic cross level mixing between the modes with different KK numbers: $(n, 0)-(m, 0),(0, n)-(0, m)$ and $(n, 0)-(0, m)$. Mixing for $n \neq m$ can be safely neglected. For $n$ odd the modes $(n, 0)-(0, n)$ cannot mix via loops as the vertexes would break the KK parity, therefore only the even modes $(n, 0)-(0, n)$ can mix and the mixing angle depends on the ratio $R_{4} / R_{5}$. In our numerical study we will focus on two limiting cases: the degenerate case $R_{4}=R_{5}$ and the decoupling limit $R_{4} \gg R_{5}$ and we describe the properties of the spectrum in each of the cases below.

\subsection{Decoupling limit $R_{4} \gg R_{5}$}

In the decoupling limit $R_{4} \gg R_{5}$, all the states $(0, n)$ and $(n, m)$, whose tree level masses have a contribution proportional to $1 / R_{5}^{2}$ decouple from the spectrum. Therefore, for our purposes, only $(n, 0)$ states will contribute. Moreover, the spectrum of the tiers is only mildly dependent on the value of $R_{5}$ via the finite contributions $\delta_{\text {finite, }}^{(n, 0)}$, therefore as a good approximation we can use the formulas for the mass corrections with $R_{4}=R_{5}$. In this limit no mixing between even modes appears. Regarding the relic abundance calculation, in fact, the potential Dark Matter candidate from $(0,1)$ is irrelevant as long as the difference in mass $1 / R_{5}-1 / R_{4}$ is or order a few times the freeze-out temperature. As the typical freezeout temperature is of order few tens of $\mathrm{GeV}$, the decoupling limit is reached even for mildly asymmetrical radii. Therefore in the decoupling limit the model will contain only one even tier, labelled by a superscript "(2)", and one odd tier, labelled by a superscript "(1)".

\subsection{Degenerate radii $R_{4}=R_{5}$}

In the symmetric $R_{4}=R_{5}=R$ case, the masses of the two tiers $(0,1)$ and $(1,0)$ are exactly degenerated: in this case we have two dark matter particles, $A^{(0,1)}$ and $A^{(1,0)}$, with exactly the same masses and spins. States in the two degenerate tires cannot annihilate each other, therefore, in the relic abundance calculation, they must be treated as independent. They can scatter via states $(1,1)$, however such processes will only contribute to the thermalisation of the Dark Matter states. This situation is true up to the presence of localised operators: in fact, adding different operators on the two singular points breaks the degeneracy and generates both mass mixing between $(1,0)$ and $(0,1)$ states and the possibility of the direct coupling of $(1,1)$ states to SM ones. In the following however we will work in the approximation where such localised operators are absent or negligible. In the relic abundance calculation we will consider a single odd tier, and multiply the final results by 2 .

The situation is different for the odd tiers: in fact, loop corrections can generate mass mixing between $(2,0)$ and $(0,2)$ states. Now the diagonal mass corrections are equal 
$\delta_{\text {finite }}^{(n, 0)}=\delta_{\text {finite }}^{(0, n)}=\delta_{\text {finite }}^{(n)}$ and the off diagonal corrections $\delta^{\prime}$ introduce a mixing between the two degenerate states. The off diagonal terms can be calculated by use of the localised counterterms [23] and they are equal to the log-divergent diagonal term [22]. As a consequence, the mass eigenstates are given by $A_{(2 \pm)} \propto A^{(2,0)} \pm A^{(0,2)}$ with the mass eigenstates equal to

$$
m_{n \pm)}^{2}=\frac{1}{R^{2}}\left(n^{2}+m_{\mathrm{SM}}^{2} R^{2}+\delta_{\text {finite }}^{(n)}(R)+n^{2} \delta_{\log } \pm \delta^{\prime}\right)
$$

with $\delta^{\prime}=n^{2} \delta_{\log }$. Therefore, the sum and difference eigenstates correspond to eigenvalues with by double or no log-divergent terms. In other words, only one of the mass eigenstates, $A^{(2+)}$, will have log-divergent contribution to the masses while the $A^{(2-)}$ will have no log-divergent contribution. Moreover, only the $A^{(2+)}$ plays a significant role in the relic abundance calculation, as it is the only one to couple to a pair of SM states via divergent loop contributions. In our model implementation, therefore, we only include this tier [22]. Therefore we can study the degenerate case by considering one odd tier and one even tier with doubled log-divergent contribution to the mass, and finally multiply by 2 the result of the relic abundance. As in the asymmetric limit the two tiers will be labelled for simplicity with the superscripts "(1)" and "(2)" respectively.

\subsection{Full numerical spectra and bound on parameters of the model}

In the following we will focus on the lightest odd tiers $(1,0)$ and $(0,1)$ and the next even tiers $(2,0)$ and $(0,2) .{ }^{2}$ As shown in the previous section the mass spectrum at loop level depends on four parameters: the two radii of extra dimensions $R_{4}$ and $R_{5}$ as well as on the cut-off scale $\Lambda$ and, for the scalar field only, on the localised mass parameter $m_{\text {loc }}$.

The radii $R_{4}$ and $R_{5}$ which set the characteristic scale of the spectrum are to be determined by the relic abundance calculation which should be compared with the available cosmological data WMAP $0.0773<\Omega h^{2}<0.1473$ [7]. On the other hand the mass splittings crucially depend on the cut-off $\Lambda$ of the effective $6 \mathrm{D}$ model which enters in the logarithmically divergent term $\delta_{\text {log }}$. Naive dimensional analysis allows us to estimate the cut off to be a few times $m_{K K}$, up to about a factor of 10 . In figure 2 we show the relative mass splitting $\Delta_{i}=\frac{m_{i}}{m_{A}(1)}-1$ with respect to the Dark Matter candidate for the odd tier as a function of $m_{K K}$. The corrections range from $\sim 0.2 \%$ for the leptons to a $\sim 10 \%$ for strongly interacting states, as it is also clear in table 2 , thus justifying the necessity to consider co-annihilation in the relic abundance calculation. The mass corrections show a mild logarithmic dependence on the cut-off, as shown in figure 3.

The $m_{\text {loc }}$ parameter is another free parameter of the model. It corresponds to the Higgs mass operator localised on the singular points of the orbifold. Note that in principle such mass term might be wrong-signed, i.e. $m_{\mathrm{loc}}^{2}<0$, and thus help trigger the electroweak symmetry breaking. This parameter plays a crucial role in the relic abundance calculation, as it enters the Higgs mass (see eq. (4.3)) and thus can change the position of the $H^{(2)}$ resonance. Effective theory order of magnitude estimates suggest that reasonable values

\footnotetext{
${ }^{2}$ The even tier $(1,1)$ may also be relevant, however it only enters in the elastic scattering of $(1,0)$ states off $(0,1)$, therefore it can be safely ignored in this discussion.
} 


\begin{tabular}{|cc|cccccccc|}
\hline$m_{K K}$ & $\Lambda R$ & $l_{R}^{(1)}$ & $l_{L}^{(1)}$ & $q_{S}^{(1)}$ & $q_{D}^{(1)}$ & $t_{S}^{(1)}$ & $t_{D}^{(1)}$ & $Z^{(1)}, W^{(1)}$ & $G^{(1)}$ \\
\hline 500 & 10 & 0.0026 & 0.0099 & 0.0458 & 0.0545 & 0.1078 & 0.1165 & 0.0373 & 0.0854 \\
500 & 5 & 0.0020 & 0.0073 & 0.0336 & 0.0399 & 0.0952 & 0.1016 & 0.0288 & 0.0602 \\
\hline 800 & 10 & 0.0039 & 0.0111 & 0.0471 & 0.0558 & 0.0751 & 0.0838 & 0.0298 & 0.0867 \\
800 & 5 & 0.0030 & 0.0083 & 0.0346 & 0.0409 & 0.0619 & 0.0682 & 0.0223 & 0.0613 \\
\hline
\end{tabular}

Table 2. Relative mass splittings $\Delta_{i}=\frac{m_{i}}{m_{A 1}}-1$ in the L1 model for two values of $m_{K K}=$ $500,800 \mathrm{GeV}$ and for two different values of the cut-off $\Lambda R=10$ and $\Lambda R=5$.

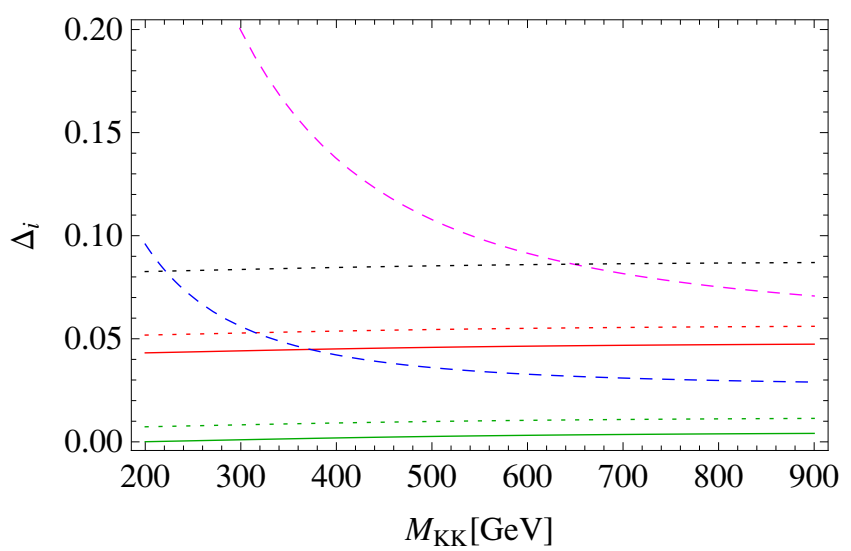

Figure 2. First KK level mass splittings as a function of $m_{K K}$ relative to the lightest state $\left(A^{(1)}\right)$. From bottom to the top: right handed leptons (green), left handed leptons (green dotted), electroweak gauge bosons (blue dashed), singlet light quarks (red), doublet light quarks (red dotted), tops (magenta dashed), gluons (black dotted).
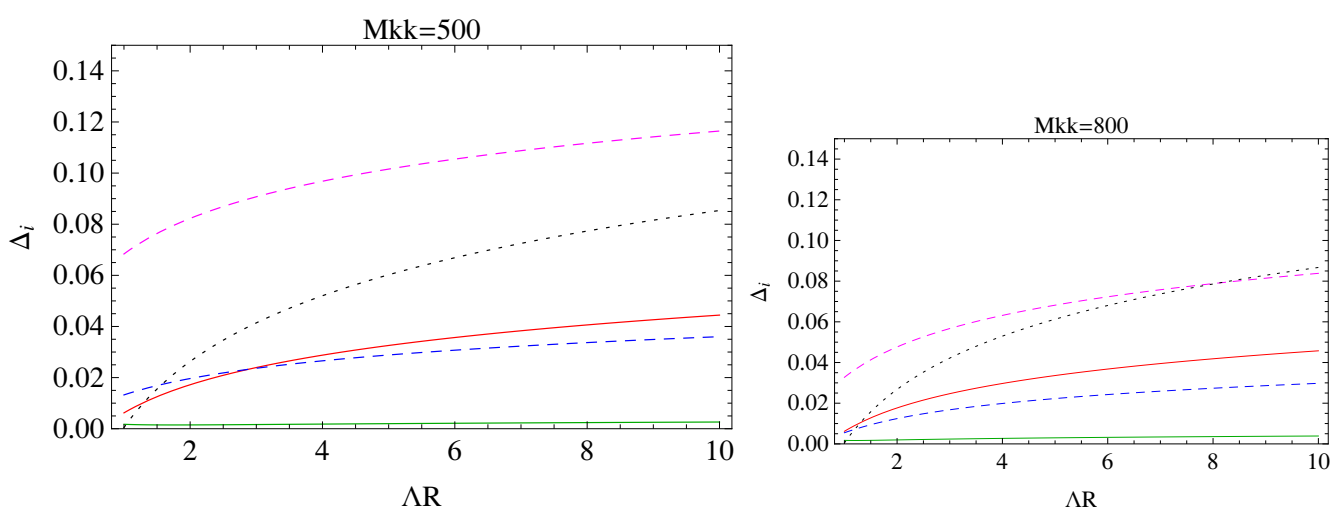

Figure 3. First KK level mass splittings as a function of $\Lambda R$. On the left panel: splittings for $m_{K K}=500 \mathrm{GeV}$, on the right panel: splittings for $m_{K K}=800 \mathrm{GeV}$. On both panels we adopt the colours from bottom to the top at $\Lambda R=10$ : leptons (green), electroweak gauge bosons (blue dashed), light quarks (red), tops (magenta dashed), gluons (black dotted). 
for the localised term should be $m_{\mathrm{loc}}<m_{K K}$. This estimate is a good starting point as it allows us to expand for small $m_{\mathrm{loc}} / m_{K K}$. It is however important to have a more precise limit on $m_{\text {loc }}$ than just the effective theory estimate $m_{\text {loc }}<m_{K K}$.

The most relevant bound on $m_{\text {loc }}$ comes from electroweak precision measurements, in particular from the $\rho$ parameter. This can be understood as follows: deriving from localised terms, $m_{\text {loc }}$ induces a mass mixing of the SM Higgs (zero mode) with all the heavy KK Higgses. In turn, after the SM Higgs boson develops a vacuum expectation value (VEV) $v$, the tadpole generated by the above mixing will propagate the VEV to each massive KK Higgs in the form

$$
<H_{(n, 0)}>=-\frac{m_{\mathrm{loc}}^{2}}{m_{K K}^{2}} \frac{v}{n^{2}}, \quad<H_{(0, m)}>=-\frac{m_{\mathrm{loc}}^{2}}{\xi^{2} m_{K K}^{2}} \frac{v}{m^{2}}, \quad<H_{(n, m)}>=-\frac{m_{\mathrm{loc}}^{2}}{m_{K K}^{2}} \frac{\sqrt{2} v}{n^{2}+\xi^{2} m^{2}} ;
$$

where $m_{K K}=1 / R_{4}$ is the mass scale related to the larger radius and $\xi=R_{4} / R_{5} \geq 1$. The bulk kinetic term of the Higgs contains quadrilinear terms between the standard Higgs boson, the higher level Higgs bosons and the corresponding $W$ 's or $Z$ 's. When these Higgses are set to the VEV, we automatically get a mass mixing between the SM $W$ and the heavy $W$ 's (and similar mixing for the SM $Z$ and the heavy $Z$ 's). These effects are not suppressed by the cut-off as we deal with $m_{\text {loc }}$ which is a dimension 6 operators in 6 dimensions: the only suppressions are due to negative powers of the heavy mass scale $m_{K K}$. These mixings will in turn correct the masses of the $W$ and $Z$, thus potentially affect the $\rho$ parameter. Other corrections are also generated to the $S$ parameter, however they turn out to be suppressed by extra powers of $m_{K K}$. The resulting correction to the $\rho$ parameter is

$$
\delta \rho=\frac{m_{W}^{2}}{m_{Z}^{2} \cos ^{2} \theta_{W}}-1=-8\left(\frac{m_{\mathrm{loc}}^{2}}{m_{K K}^{2}}\right)^{2} \frac{m_{Z}^{2}-m_{W}^{2}}{m_{K K}^{2}} f(\xi)
$$

where $\xi=\frac{R_{4}}{R_{5}}$ and $f(\xi)$ is a number of order 1 , given by the function

$$
f(\xi)=\frac{\pi^{6}}{945} \frac{\xi^{6}+1}{\xi^{6}}+2 \sum_{n, m=1}^{\infty} \frac{1}{\left(n^{2}+\xi^{2} m^{2}\right)^{3}} .
$$

Numerically, $f(\xi)$ smoothly decreases with increasing $\xi$ and varies from $f(1)=2.33$ to $f(\infty)=1.017$. We finally compare the correction $\delta \rho$ (which is negative in this model) to the value given in PDG $\rho=1.0004_{-0.0004}^{+0.0003}$ and obtain the corresponding bounds in the $m_{\text {loc }} / m_{K K}$ plane at $3 \sigma$, which are shown in figure 4 . Note that the bound is independent on the sign of $m_{\text {loc }}^{2}$.

The localised counter-terms, that encode the divergent loop corrections to the mass, generate additional corrections to the $\rho$ parameter: for instance log divergent mass corrections to the Higgs will generate a similar contribution to the $\rho$ parameter, while corrections to the Higgs kinetic terms can generate mixing between light and heavy gauge bosons via the SM Higgs VEV. Such corrections are suppressed by a loop factor compared to $m_{\text {loc }}$, however they may be numerically relevant for the extraction of a precise bound on $m_{\text {loc }}$. This task would however require a full one-loop study of the corrections to electroweak precision measurements, which is beyond the scope of this paper. In this section, we will 


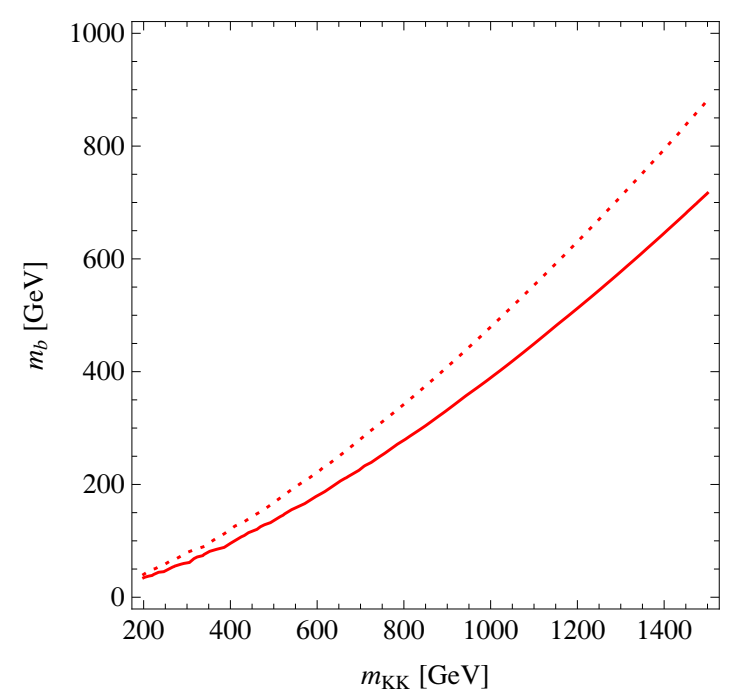

Figure 4. Bound on $m_{\mathrm{loc}}$ as a function of the $m_{K K}$ mass, obtained imposing that the effect on the $\rho$ parameter is not larger than the allowed measured value within 3 sigmas. Plain line correspond to the bound in the symmetric scenario $R_{4}=R_{5}$, while dashed line to the asymmetric case $R_{4} \gg R_{5}$.

take the bound on $m_{\mathrm{loc}}$ as an indicative value, assuming that there will be no important cancellations with the loop-induced corrections.

Full spectrum for the first and second tiers. In tables 3 and 4 we give values of masses of all the particles present in the model for three benchmark points which will be important for the phenomenological analysis: $m_{K K}=300,500,800 \mathrm{GeV}$. The radiative corrections to the KK masses depend on whether we assume $R_{4}=R_{5}$ or $R_{4} \gg R_{5}$ only in the second tier $(2,0)-(0,2)$ as there are cross-level mixing terms in this case. We give the mass spectrum in both cases.

In the first tier the lightest state is always $A^{(1)}$, which is a neutral spin-0 particle and corresponds to the Dark Matter candidate in our model. For $m_{K K}<200 \mathrm{GeV}$ the lightest particle in the first tier is the singlet electron which excludes the model in this range of $m_{K K} \cdot A^{(1)}$ is the KK resonance of the photon, however the mixing angle between $\mathrm{SU}(2)$ and $\mathrm{U}(1)$ components is different from the SM one. In particular, it is smaller than the Weinberg angle and, for large $m_{K K}$, the DM candidate is predominantly a U(1) gauge boson. In the degenerate case, two such particles with the same mass exist while in the decoupling limit only the lightest one will significantly contribute to the Dark Matter abundance. The next lightest particles are singlet and doublet leptons and the SU(2) gauge bosons which will play an important role in the dark matter phenomenology in the co-annihilation processes. Coloured particles are heavier but still their role cannot be neglected in the dark matter prediction and in collider phenomenology as they have strong couplings which will enhance all the processes where quarks are involved. Note that there may be additional contributions from the cut-off scale physics to operators localised at the two singular points of the fundamental domain. In principle, these could turn some other 


\begin{tabular}{|c|c|c|c|}
\hline$m_{K K}[\mathrm{GeV}]$ & 300 & 500 & 800 \\
\hline$A^{(1)}$ & 300.9 & 500.5 & 800.2 \\
$l_{S}^{(1)}$ & 301.3 & 501.5 & 803.0 \\
$l_{D}^{(1)}$ & 303.4 & 504.5 & 808.4 \\
$d_{S}^{(1)}$ & 313.8 & 519.2 & 836.6 \\
$u_{S}^{(1)}$ & 314.2 & 519.7 & 837.6 \\
$W^{(1)}$ & 317.8 & 515.0 & 822.1 \\
$Z^{(1)}$ & 319.6 & 515.8 & 822.3 \\
$q_{D}^{(1)}$ & 316.8 & 522.9 & 843.9 \\
$t_{S}^{(1)}$ & 361.0 & 550.5 & 859.8 \\
$t_{D}^{(1)}$ & 363.6 & 553.6 & 866.1 \\
$G^{(1)}$ & 326.1 & 534.9 & 868.4 \\
\hline
\end{tabular}

Table 3. Typical masses of the particles of the level (1) at $m_{K K}=300,500$ and $800 \mathrm{GeV}$. The mass splittings are independent on the symmetric or asymmetric case as there is no cross-level mixing.

\begin{tabular}{|c|c|c|c|c|c|c|c|}
\hline$m_{K K}[\mathrm{GeV}]$ & 300 & 500 & 800 & $m_{K K}[\mathrm{GeV}]$ & 300 & 500 & 800 \\
\hline$S^{(2)}$ & 600.0 & 993.4 & 1568.3 & $S^{(2)}$ & 573.3 & 955.6 & 1528.9 \\
$A^{(2)}$ & 600.9 & 1000.3 & 1599.8 & $A^{(2)}$ & 601.0 & 1000.2 & 1599.5 \\
$H^{(2)}$ & 600.0 & 1001.3 & 1573.3 & $H^{(2)}$ & 586.8 & 963.7 & 1534.0 \\
$l_{S}^{(2)}$ & 602.3 & 1001.2 & 1605.4 & $l_{S}^{(2)}$ & 604.5 & 1007.5 & 1612.0 \\
$l_{D}^{(2)}$ & 606.2 & 1003.2 & 1615.0 & $l_{D}^{(2)}$ & 612.3 & 1020.6 & 1632.9 \\
$W^{(2)}$ & 619.6 & 1010.5 & 1636.8 & $W^{(2)}$ & 633.5 & 1050.5 & 1677.9 \\
$Z^{(2)}$ & 620.0 & 1010.8 & 1636.8 \\
$d_{S}^{(2)}$ & 625.0 & 1014.7 & 1665.5 & $Z^{(2)}$ & 633.8 & 1050.6 & 1677.9 \\
$u_{S}^{(2)}$ & 625.7 & 1015.0 & 1667.3 & $d_{S}^{(2)}$ & 659.9 & 1094.4 & 1744.0 \\
$q_{D}^{(2)}$ & 630.4 & 1017.5 & 1678.6 & $u_{S}^{(2)}$ & 661.4 & 1096.9 & 1748.0 \\
$t_{S}^{(2)}$ & 651.9 & 1031.3 & 1682.0 & $q_{D}^{(2)}$ & 670.7 & 1112.5 & 1772.9 \\
$G^{(2)}$ & 653.1 & 1031.8 & 1739.2 & $t^{(2)}$ & 688.4 & 1118.2 & 1768.7 \\
$t_{D}^{(2)}$ & 656.6 & 1033.7 & 1693.3 \\
$G^{(2)}$ & $t_{D}^{(2)}$ & 621.1 & 1191.8 & 1893.5 \\
\hline
\end{tabular}

Table 4. Typical masses of the particles of the level (2) at $m_{K K}=300,500$ and $800 \mathrm{GeV}$. On the left the non-symmetric case $R_{4} \gg R_{5}$. On the right the symmetric case $R_{4}=R_{5}$. In both cases, for the Higgses $m_{\mathrm{loc}}=0$.

particle into the lightest KK-odd state. Hence, the odd modes of neutrinos $\nu^{(1)}$ or the neutral electroweak gauge boson $Z^{(1)}$ could all be viable dark matter candidates too. We leave the investigation of these possibilities for future work. 

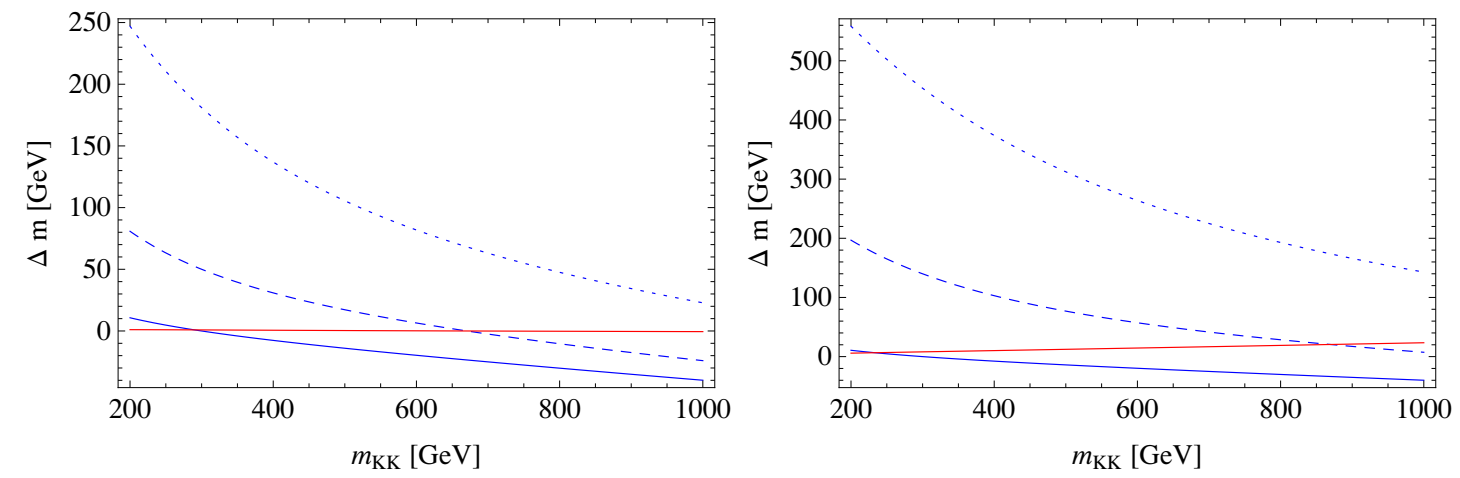

Figure 5. Mass splitting $\Delta m=m-m_{\text {tree }}$ in the Higgs sector in tier (2). We show the splittings of $H^{(2)}$ for three values of $m_{\mathrm{loc}}$ parameter: $m_{\mathrm{loc}}=0 \mathrm{GeV}$ in blue, $m_{\mathrm{loc}}=250 \mathrm{GeV}$ in blue dashed and $m_{\text {loc }}=500 \mathrm{GeV}$ in blue dotted. On the left non-symmetric case $R_{4} \gg R_{5}$. On the right symmetric case $R_{4}=R_{5}$. Red line corresponds to the $A^{(2)}$ splitting for comparison.

In the second tier, the lightest particle is a vector gauge boson $A^{(2)}$ and Higgs excitations $H^{(2)}$ and $S_{ \pm, 0}^{(2)}$, if the localised mass parameter $m_{\mathrm{loc}}$ is very small or $m_{\text {loc }}^{2}<0$. The dependence of the Higgs resonance masses to $m_{\text {loc }}$ is shown in figure 5 . Due to the extremely small mass corrections, $A^{(2)}$ can only decay into SM particles via loop induced interactions and thus will play an important role in the Dark Matter and LHC phenomenology enhancing the resonant productions of SM particles. The weak gauge bosons $W^{(2)}$ and $Z^{(2)}$ will decay into other heavy particles but the resonant decays into SM fermions will not be negligible as well. All the level (2) particles will also participate as final states of the annihilations and co-annihilations of the primordial cosmic plasma reducing significantly the relic abundance of the dark matter and thus changing strongly the bounds of the cosmologically allowed $m_{K K}$ values [26]. We will present those phenomenological aspects of the model in the following sections.

Note that, in contrast to the chiral square [24, 25] and to the 5D mUED $[27,28]$ models where the odd level Higgs boson is also a viable dark matter candidate in some parameter space, the Higgs boson of level $(1,0)$ and $(0,1)$ mode is not present on the $\mathrm{RP}^{2}$. In the following, we will neglect the contributions of the localised operators under the assumption that their contribution is smaller that the loop ones. We will first discuss some features of the two limiting cases under study and the numerical results for the spectra before turning our attention to the calculation of the relic abundance.

\section{Analytical results}

In our computation we consider a general particle spectrum without any simplifying assumptions. In particular we do not assume a completely degenerate particle spectrum but we keep the KK masses after one-loop corrections to the (1) modes (the analytic formulas, to simplify the notation, will be however shown assuming the masses at each KK level degenerate: i.e. for the first level particles (1) we assume $m_{X^{(1)}}=m_{A^{(1)}}$ and for the second level particles (2) the mass will be $m_{X^{(2)}}=2 m_{A^{(1)}}$ for every particle $X$ in a given tier). We 


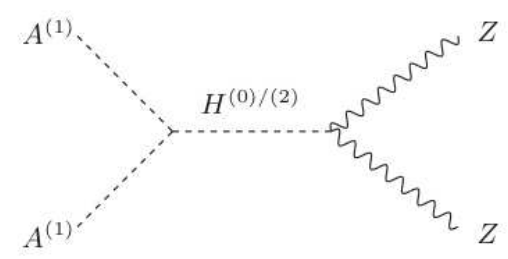

Figure 6. Annihilations of $A^{(1)} A^{(1)}$ into Standard Model $Z$ gauge bosons.

keep all the SM particle masses non zero, except the electron mass which will be neglected. Moreover we do not neglect the mixing between the $\mathrm{U}(1) B^{(1)}$ and $\mathrm{SU}(2) W_{3}^{(1)}$ components, which originates from the electroweak symmetry breaking and is expected to be small for large $m_{K K}$ but effectively is important for small values of $m_{K K}$.

In our analytic and numerical calculations we make some simplifying assumptions for the Yukawa couplings. We neglect all the Yukawa couplings, which are proportional to the corresponding fermion mass, for all the light SM particles except the top quark. This assumption is well justified as the top Yukawa coupling can alter significantly the cross section result as it leads to resonant s-channel diagrams. Those resonant effects are important only for the exchange of the (2) level particles and as we will see, they enhance the effective cross section in a small region of $m_{K K}$ near the resonant value. Aside those effects, we do not expect our results to be sensitive to the masses of light fermions.

In all the analytic calculations when examining the relic density as a function of mass $m_{K K}$ we neglect the mass dependence of $x_{F}$. The value of $x_{F}$ depends weakly on the mass. This dependence is approximately logarithmic. Typically, over the mass range $m_{K K}=$ $0.2-1 \mathrm{TeV}, x_{F}$ varies by about $0.1 \mathrm{GeV} /$ degree, or less than $15 \%$. This variation has small effects on the relic density. This also shows that the dark matter is cold. In all cases considered here we obtain $22 \lesssim x_{F} \lesssim 30 \mathrm{GeV}$, so that the particles are well approximated as non-relativistic. This implies freeze-out temperatures in the range $34 \div 45 \mathrm{GeV}$ depending on the scenario.

\subsection{LKP annihilations - analytic expressions for the cross sections}

In this section we show the calculation of annihilation cross section in details in order to fix the notation. The dark matter candidate can annihilate into all SM particles. As we will see the cross sections into SM gauge bosons give the leading contributions. The fermionic final states will not contribute once we develop the cross section for small velocities.

\subsection{1 $A^{(1)} A^{(1)} \rightarrow Z Z$}

The annihilation $A^{(1)}$ into $Z$ gauge bosons is mediated only by the SM Higgs exchange presented in figure 6. The interaction of the $A^{(1)}$ with the Standard Model Higgs boson $h$ is given by

$$
\mathcal{L}_{h}^{4 D}=-\frac{g_{2}^{2}}{2} \frac{\left(c_{w} s_{w 1}-c_{w 1} s_{w}\right)^{2}}{c_{w}^{2}} A^{(1)} A^{(1)} h(h+v)
$$




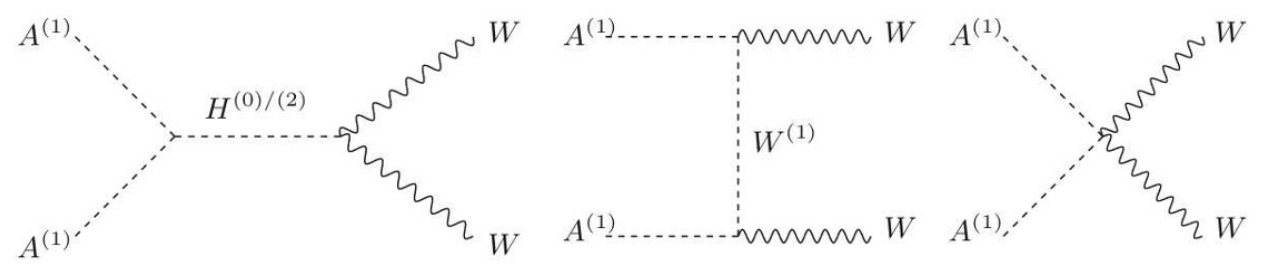

Figure 7. Annihilations of $A^{(1)} A^{(1)}$ into SM W gauge bosons.

where $g_{2}$ is the $\mathrm{SU}(2)$ gauge coupling constant, $s_{w}$ and $c_{w}$ are the sine and cosine of the Standard Model Weinberg mixing angle $\left(s_{w}^{2}=0.23\right)$ and $s_{w 1}, c_{w 1}$ are the electroweak mixing angles in the first KK tier and $v \approx 246 \mathrm{GeV}$ is the electroweak scale. The annihilation cross section into a pair of $Z$ bosons reads

$$
\sigma\left(A^{(1)} A^{(1)} \rightarrow Z Z\right)=Y_{A^{1}}^{2} Y_{Z}^{2} \frac{s^{2}-4 s m_{Z}^{2}+12 m_{z}^{4}}{128 \pi s m_{Z}^{4}\left(s-m_{h}^{2}\right)^{2}} \sqrt{\frac{s-4 m_{Z}^{2}}{s-4 m_{A^{1}}^{2}}}
$$

where $Y_{A^{1}}=\frac{g_{2}^{2} v\left(c_{w} s_{w 1}-c_{w 1} s_{w}\right)^{2}}{2 c_{w}^{2}}$ is the Higgs- $A^{(1)}$ coupling and $Y_{Z}=\frac{g_{2}^{2} v}{2 c_{w}^{2}}$ is the SM Higgs- $Z$ coupling constant. Expanding the cross section in powers of the relative speed $v_{\text {rel }}$ between the $A^{(1)}$ photons gives

$$
v_{\mathrm{rel}} \sigma\left(A^{(1)} A^{(1)} \rightarrow Z Z\right) \approx a_{Z Z}+b_{Z Z} v_{\mathrm{rel}}^{2}+\mathcal{O}\left(v_{\mathrm{rel}}^{4}\right)
$$

and the first two terms in this non-relativistic expansion are

$$
a_{Z Z}=Y_{A^{1}}^{2} Y_{Z}^{2} \frac{4 m_{A^{1}}^{4}-4 m_{A^{1}}^{2} m_{Z}^{2}+3 m_{Z}^{4} \sqrt{m_{A^{1}}^{2}-m_{Z}^{2}}}{64 \pi m_{A^{1}}^{3} m_{Z}^{4}\left(m_{h}^{2}-4 m_{A^{1}}^{2}\right)^{2}}
$$

and

$$
\begin{aligned}
& b_{Z Z}=-Y_{A^{1}}^{2} Y_{Z}^{2} \times \\
& \frac{64 m_{A^{1}}^{8}-176 m_{A^{1}}^{6} m_{Z}^{2}+4 m_{A^{1}}^{4}\left(3 m_{h}^{2} m_{Z}^{2}+52 m_{Z}^{4}\right)-12 m_{A^{1}}^{2}\left(2 m_{h}^{2} m_{Z}^{4}+9 m_{Z}^{6}\right)+15 m_{h}^{2} m_{Z}^{6}}{512 \pi m_{Z}^{4}\left(4 m_{A^{1}}^{3}-m_{A^{1}} m_{h}^{2}\right)^{3} \sqrt{m_{A^{1}}^{2}-m_{Z}^{2}}} .
\end{aligned}
$$

\subsection{2 $\quad A^{(1)} A^{(1)} \rightarrow W^{+} W^{-}$}

The annihilation of $A^{(1)}$ into $W^{ \pm}$gauge bosons is mediated by the SM Higgs exchange in the $s$-channel, by the $W^{(1)}$ scalar partners of $W$ in $t$ and $u$-channels and finally by a direct quartic coupling with two Standard Model $W^{ \pm}$gauge bosons (see figure 7 ). For the annihilation cross section we obtain: (here for simplicity we give the results with all the SM masses neglected and for the degenerated KK masses, but the full results are retained 

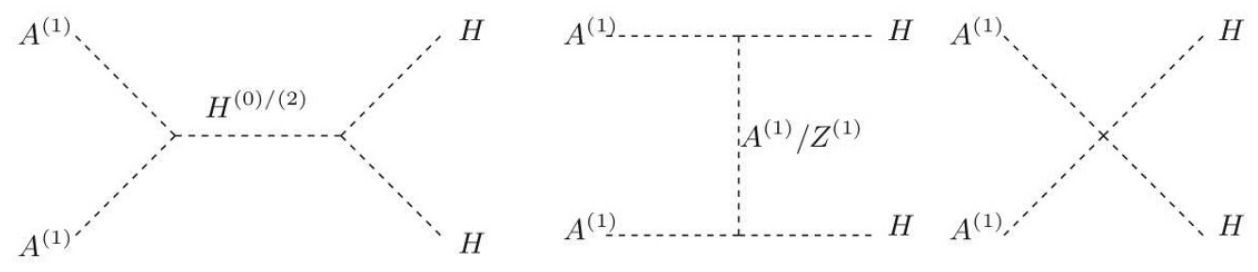

Figure 8. Annihilations of $A^{(1)}$ into SM Higgs bosons.

in the numerical analysis)

$$
\begin{aligned}
\sigma\left(A^{(1)} A^{(1)} \rightarrow W^{+} W^{-}\right)= & \frac{g_{2} s_{w 1}}{2 \pi s\left(s-4 m_{A^{1}}^{2}\right)} \times \\
& {\left[\frac{s^{3}-16 s m_{A^{1}}^{4}}{\sqrt{s\left(s-4 m_{A^{1}}^{2}\right)}}-4 m_{A^{1}}^{2}\left(s-2 m_{A^{1}}^{2}\right) \ln \frac{s+\sqrt{s\left(s-4 m_{A^{1}}^{2}\right)}}{s-\sqrt{s\left(s-4 m_{A^{1}}^{2}\right)}}\right], }
\end{aligned}
$$

and the first two coefficients of the non-relativistic expansion are

$$
\begin{aligned}
& a_{W W}=\frac{g_{2}^{4} s_{w 1}^{4}}{4 \pi m_{A^{1}}^{2}}, \\
& b_{W W}=-\frac{5}{6} a_{W W} .
\end{aligned}
$$

\subsection{3 $\quad A^{(1)} A^{(1)} \rightarrow h h$}

Finally for the Higgs boson production (figure 8), neglecting all the SM masses, we get

$$
\sigma\left(A^{(1)} A^{(1)} \rightarrow h h\right)=\frac{g_{w}^{4}\left(c_{w 1} s_{w}-c_{w} s_{w 1}\right)^{4}}{128 \pi c_{w}^{4} \sqrt{s\left(s-4 m_{A^{1}}^{2}\right)}},
$$

and the non-relativistic coefficients read:

$$
\begin{aligned}
a_{h h} & =\frac{g_{w}^{4}\left(c_{w 1} s_{w}-c_{w} s_{w 1}\right)^{4}}{256 \pi c_{w}^{4} m_{A^{1}}^{2}}, \\
b_{h h} & =-\frac{1}{2} a_{h h} .
\end{aligned}
$$

\subsection{4 $A^{(1)} A^{(1)} \rightarrow f \bar{f}$}

The annihilation of $A^{(1)}$ into light fermionic degrees of freedom $f$ (quarks $u, d, s, c, b$ and all the leptons) is mediated through the exchange of level one singlet and doublet fermions $f_{D / S}^{1}$ into $t$ and $u$ channels, see figure 9. Moreover we include the Yukawa couplings of the top quark $t \bar{t} h$, thus the annihilation into two top quarks will have additional contribution form the SM Higgs in the $s$-channel. The production of neutrinos is mediated only by the exchange of doublet neutrinos $\nu_{D}^{(1)}$ in $t$ and $u$ channel. The coefficients $a_{F F}$ and $b_{F F}$ are both proportional to the SM fermion mass. In the first approximation they will give both 


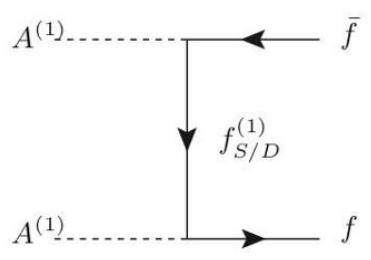

Figure 9. Annihilations of $A^{1} A^{1}$ into light SM fermions.

\begin{tabular}{|c|c|}
\hline$f_{S}$ & $q_{f} A$ \\
$l_{D}$ & $\frac{1}{2}(A+B)$ \\
$\nu_{D}$ & $\frac{1}{2}(A-B)$ \\
$u_{D}$ & $\frac{1}{6} A+\frac{1}{2} B$ \\
$d_{D}$ & $\frac{1}{6} A-\frac{1}{2} B$ \\
\hline
\end{tabular}

Table 5. Couplings of $A^{(1)}$ with fermions.

a zero result, therefore we give their expressions without neglecting the SM masses but assuming degenerated KK spectrum for simplicity:

$$
\begin{aligned}
& a_{F F}=\left(Y_{D}^{2}+Y_{S}^{2}\right)^{2} \frac{m_{q}^{2}\left(m_{A^{1}}^{2}-m_{q}^{2}\right)^{3 / 2}}{8 \pi m_{A^{1}}^{3}\left(m_{q}^{2}-2 m_{A^{1}}^{2}\right)^{2}}, \\
& b_{F F}=-\left(Y_{D}^{2}+Y_{S}^{2}\right)^{2} \frac{m_{q}^{2} \sqrt{m_{A^{1}}^{2}-m_{q}^{2}}\left(72 m_{A^{1}}^{6}-148 m_{A^{1}}^{4} m_{q}^{2}+82 m_{A^{1}}^{2} m_{q}^{4}-15 m_{q}^{6}\right)}{192 \pi m^{3}\left(m_{q}^{2}-2 m_{A^{1}}\right)^{4}},
\end{aligned}
$$

where $m_{q}$ is the outgoing quark mass and $Y_{S / D}$ are the couplings $f f_{S / D}^{(1)} A^{1}$ defined in table 5 in terms of $A=\frac{g_{2} s_{w} c_{w 1}}{c_{w}}, B=\frac{g_{2} c_{w} s_{w 1}}{c_{w}}$ and the fermion electric charge $q_{f}$. For the top quarks one has to include the Yukawa mixings between singlet and doublet states by multiplying the corresponding constants by the top mixing angle $\cos \alpha$ for $t_{S}^{(1)}$ and $\sin \alpha$ for $t_{D}^{(1)}$. The neutrino production cross section expansion will then simply vanish. The top quarks production coefficients will have additional contributions from the $s$-channel Higgs exchange.

$$
\begin{aligned}
& a_{T T}=a_{F F}+\frac{g_{2}^{4}\left(c_{w} s_{w 1}-c_{w 1} s_{w}\right)^{4}}{4 c_{w}^{4}} \frac{m_{q}^{2} \sqrt{m_{A^{1}}^{2}-m_{q}^{2}}}{8 \pi m_{A^{1}}\left(m_{h}^{2}-4 m_{A^{1}}^{2}\right)^{2}}, \\
& b_{T T}=b_{F F}-\frac{g_{2}^{4}\left(c_{w} s_{w 1}-c_{w 1} s_{w}\right)^{4}}{4 c_{w}^{4}} \frac{m_{q}^{2}\left(24 m_{A^{1}}^{4}-2 m_{A^{1}}^{2}\left(m_{h}^{2}+14 m_{q}^{2}\right)+3 m_{h}^{2} m_{f}^{2}\right)}{64 \pi m_{A^{1}}\left(m_{h}^{2}-4 m_{A^{1}}^{2}\right)^{2} \sqrt{m_{A^{1}}^{2}-m_{q}^{2}}} .
\end{aligned}
$$

\subsection{Influence of KK mass degeneracy and Higgs contribution}

In this section we will carefully study the influence of various effects on the relic abundance of Dark Matter: 


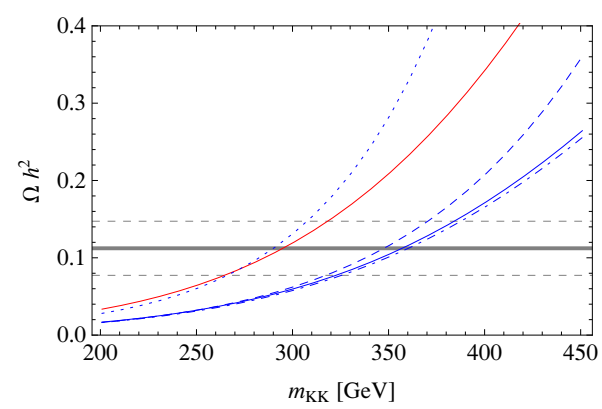

Figure 10. Analytic result of the relic abundance calculation from annihilations only on the real projective plain. In these plots we show the impact of several factors on the relic abundance: we start with the simplest case (blue dotted line) where we assume equal masses for the first KK level and no SM Higgs exchange in $s$ channel. Then progressively we add the contributions: the (1) level mass corrections (blue dotted), Higgs $s$-channel contribution (blue dot-dashed), relativistic corrections (blue solid line). We add also the same result assuming the symmetric radii $R_{4}=R_{5}$ with all the above corrections included (red solid line).

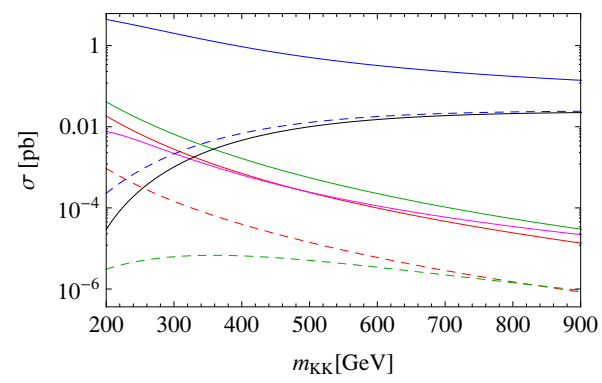

Figure 11. Averaged annihilation cross sections $\sigma\left(A^{(1)} A^{(1)} \rightarrow S M\right)$ of the LKP into Standard Model final states. In red (red-dashed) up (down) type quarks, magenta - tops, green (greendashed) - charged leptons (neutrinos), blue (blue dashed) - WW (ZZ) gauge bosons, in black - HH. The cross section is calculated at $p_{c m s}=100 \mathrm{GeV}$ (consistent for non-relativistic DM, the maximum of the Boltzmann distribution is given by $p_{0}=\sqrt{2 m k T}=m \sqrt{2 k / x_{F}} \approx 0.28 m_{K K}$ for $k=1, x_{F}=25$ and vary between $56-280 \mathrm{GeV}$ for $\left.m_{K K}=200-1000 \mathrm{GeV}\right)$.

1. degeneracy of masses in the KK tiers,

2. SM Higgs exchange,

3. relativistic corrections,

using analytic expressions for cross sections and relic abundance. The results are shown in figure 10. We also summarise the allowed values of $m_{K K}$ in table 6 where we give the preferred ranges for the decoupling limit $R_{4} \gg R_{5}$ on the left and for the symmetric degenerate radii $R_{4}=R_{5}$ in the right column.

We first consider only annihilation processes: the average annihilation cross sections as a function of the KK mass are shown in figure 11. 


\begin{tabular}{|c|c|c|}
\hline & $R_{4} \gg R_{5}$ & $R_{4}=R_{5}$ \\
\hline equal masses & $266-307$ & $221-262$ \\
corrected masses & $317-370$ & $263-313$ \\
+ s-channel Higgs & $325-388$ & $267-321$ \\
+ relativistic corrections & $322-384$ & $264-318$ \\
\hline
\end{tabular}

Table 6. Preferred ranges for $m_{K K}$ (in $\mathrm{GeV}$ ) from the analytic relic abundance calculation. In the first approximation we include only annihilations of LKP and study the impact of including the corrections 1) non-degenerated spectrum of the first KK level (line 2), 2) including $s$-channel SM Higgs exchange (line 3), including relativistic correction to the $b$-rel coefficient (line 4). In the first line we show the bounds obtained for the simplest case where all the SM masses are neglected and the KK spectrum is fully degenerate. In the left column we present the results for the non-degenerated radii $R_{4} \gg R_{5}$, in the right column - for the symmetric radii $R_{4}=R_{5}$.

The blue dotted line in figure 10 presents the relic abundance assuming all the (1) level KK states have the same mass and the Higgs couplings are neglected as well. In this regime only annihilations into the $W$ gauge bosons contribute considerably. Annihilations into $Z$ and Higgs bosons are turned off and the fermion production is negligible (see figure 11). Then we release the assumption of the degeneracy of the KK states (blue dashed line) and use the full one loop spectrum: the relic abundance is considerably reduced. If we take a representative point $m_{K K}=350 \mathrm{GeV}$, which lies in the range allowed by WMAP7 data (for the most complete scenario) we obtain $\Omega h^{2}=0.285$ for the equal mass scenario, a value far above the experimental data, which is pushed down to $\Omega h^{2}=0.121$ for the exact spectrum - a value which satisfies the experimental bounds. This enormous reduction of about $60 \%$ is due to the considerable changes in the annihilation cross section into $W$ bosons.

As dealing with large analytic formulas is difficult, in figure 11 we compare the first coefficient of the non-relativistic expansion $a_{\text {rel }}$ for the three cases studied here. First note that effectively the contributions coming from the annihilations into quark and leptons are almost negligible when compared to the annihilations into gauge bosons which are two orders of magnitude larger. Then when we release the equal mass approximation, as the masses of $W^{(1)}$ scalars exchanged in the $t$ and $u$ channels appear in the numerator and denominator it is difficult to guess what will be the overall impact of the mass degeneracy. But from the plot we see that in the equal mass approximation, the value of the annihilation cross section $\sigma\left(A^{(1)} A^{(1)} \rightarrow W^{+} W^{-}\right)$is significantly reduced: at the sample point $m_{K K}=$ $350 \mathrm{GeV}$ we find a ratio $a_{W W}^{\text {equal }} / a_{W W}^{\text {exact }}=0.376$. The behaviour of the fermion production is completely different. The result is shown on the right panel of figure 12 . We sum the annihilations into all fermionic degrees of freedom and show the first coefficient of the non-relativistic expansion for the equal mass spectrum of the first KK level with the red dotted line and for the non-degenerated spectrum with the red dashed line. In this case, in contrast to the annihilations into gauge bosons $a_{\text {rel }}$, we see that the degeneracy of the spectrum will enhance the annihilation cross section $\sigma\left(A^{(1)} A^{(1)} \rightarrow f \bar{f}\right)$. Analytically this is simple to explain as the masses of the first level quarks $q_{D / S}^{(1)}$ exchanged into $t$ and $u$ channels cancel in the numerators while taking the trace of the $\mathcal{S}$-matrix element. We 

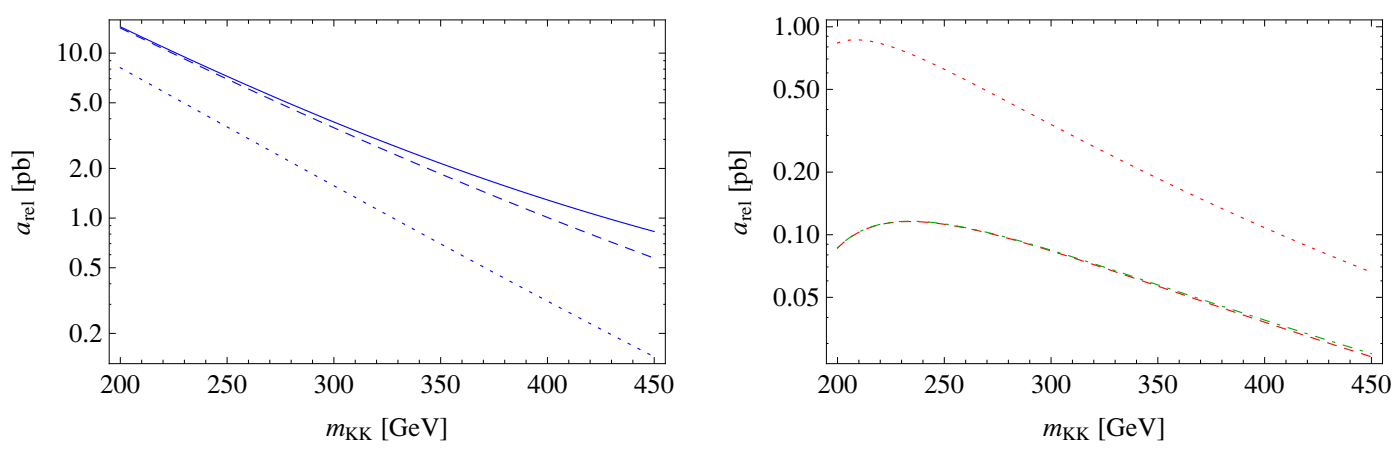

Figure 12. First coefficients of the non relativistic expansion $\left\langle\sigma v_{\text {rel }}\right\rangle \approx a_{\text {rel }}+v_{\text {rel }}^{2} b_{\text {rel }}$. On the left panel the coefficients of the annihilations into gauge boson are shown, in blue dotted - for equal $K K$ masses, in blue dashed - exact $K K$ masses, in blue dot dashed line - the Higgs contribution. On the right panel - coefficients of the annihilations into all fermions summed. In red dotted equal $m_{(1)}$ masses, in red dashed - exact $m_{(1)}$ masses and in green we add the Higgs contribution.

are then left with the $m_{q^{(1)}}$ into denominators only. Schematically we can write that the annihilation cross section into fermions is proportional to

$$
\sigma \sim \frac{1}{\left(m_{1}^{2}+m_{D / S}^{2}-m_{q}^{2}\right)^{2}} \approx \frac{1}{\left(m_{1}^{2}+m_{D / S}^{2}\right)^{2}}=\frac{1}{m_{1}^{4}\left(1+x^{2}\right)^{2}} .
$$

As the quarks receive large loop corrections to the masses, for $m_{K K}=350 \mathrm{GeV}$ we have $x=\frac{m_{D}}{m_{1}}=1.17$ for the $t_{D}^{(1)}$ top quark we obtain

$$
\frac{\sigma_{\text {exact }}}{\sigma_{\text {equal }}} \approx \frac{4}{\left(1+x^{2}\right)^{2}}=0.71 .
$$

Thus effectively the annihilation cross section into fermions is overestimated if one assumes degenerate KK spectrum. Contributions coming from quarks being considerably smaller than the gauge boson contribution, the enhancement of the bosonic cross section wins and we observe the decrease of the relic abundance.

Next we add the $s$-channel Higgs exchange, blue dot-dashed line in figure 10. The effect is small but visible. As we add new channels to the annihilation cross section the relic abundance is reduced. The modification is of about $13 \%$ compared to the previous case where we have considered the non-degenerate KK spectrum without Higgs contributions. Note that now all the annihilation channels contribute, that is the cross sections $\sigma\left(A^{(1)} A^{(1)} \rightarrow Z Z, h h\right)$ are present. The right panel on figure 13 shows the relative contributions of different final states into the total annihilation cross section. The $W^{+} W^{-}$ gauge boson production still gives the leading contribution, but we can observe the growing contributions of $Z Z$ and $h h$ final states. What is worth noticing is that while we do not neglect the Standard Model masses we effectively get new contributions to the total annihilation cross section coming from fermionic, $Z Z$ and $h h$ final states. From this analysis we see that these processes cannot simply be neglected as their contribution changes of about $13 \%$ the relic abundance prediction. 

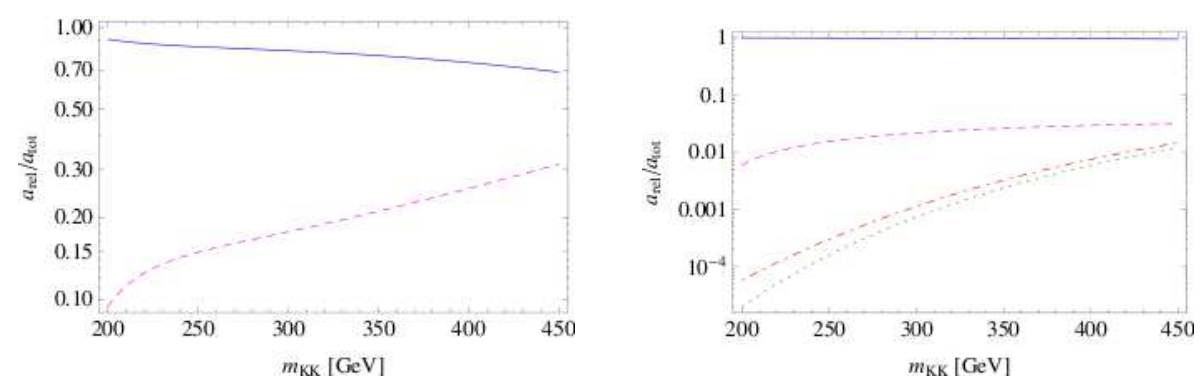

Figure 13. On the left: relative contribution of WW annihilation (blue line) and all fermion annihilations (dashed magenta) for the equal mass spectrum. On the right: relative contributions $a_{X X} / a_{\text {tot }}$ for the exact spectrum and Higgs channel added. In blue $W W$, in dotted green $h h$, in red dot-dashed $Z Z$, in magenta dashed $-f \bar{f}$ final states.

\section{Relic abundance - numerical results}

We now turn to studying the $\mathrm{RP}^{2}$ model numerically. In the following we estimate the WMAP preferred range of the compactification scale $m_{K K}$ of the extra dimensions using the relic abundance calculation performed numerically with MicrOMEGAs [5, 6]. The general assumptions are the same as for the analytic calculations, presented at the beginning of section 5 .

First we have implemented the model into FeynRules [29] where all the effective Lagrangians of the model in terms of 4-dimensional fields and couplings are given in Mathematica language and interfaced with Monte Carlo generators like CalcHEP [30]. We have implemented the full set of states in tiers (1) and (2) including all bulk couplings and one-loop order masses. We also implemented the loop induced couplings that mediate the decays of the even states into a pair of SM states: the coefficients are calculated in [23] using the effective counter-terms in the "magic gauge" $\xi=-3$, however they are only valid for on-shell external particles. Therefore, the loop induced couplings can only be used consistently to compute decay widths or processes with a resonant exchange of a (2) state. A full implementation would require the inclusion of gauge-dependent loop corrections to all vertexes, including bulk ones, and is beyond the scope of this work. We also modified the implementation in order to reproduce the symmetric radii case $\left(R_{4}=R_{5}\right)$, in which case the loop corrections to the masses of the even states and their loop induced couplings are corrected by a factor of 2 and $\sqrt{2}$ respectively. The presence of two degenerate odd tiers is taken into account by doubling the final result of the relic abundance. The CalcHEP output from FeynRules was then incorporated into MicrOMEGAs, and we validated the numerical implementation against the analytic results of the previous sections. Unless otherwise stated, the free parameters of the model are fixed to $m_{\mathrm{loc}}=0$ and $\Lambda R=10$, while the SM Higgs mass is taken to be $125 \mathrm{GeV}$. The WMAP7 bound on the relic density is $0.0773<\Omega h^{2}<0.1473$. We calculate the relic abundance using the implementation of the model to MicrOMEGAs v2.4.1. We study two main cases, in order to understand the impact of various effects on the relic abundance: 

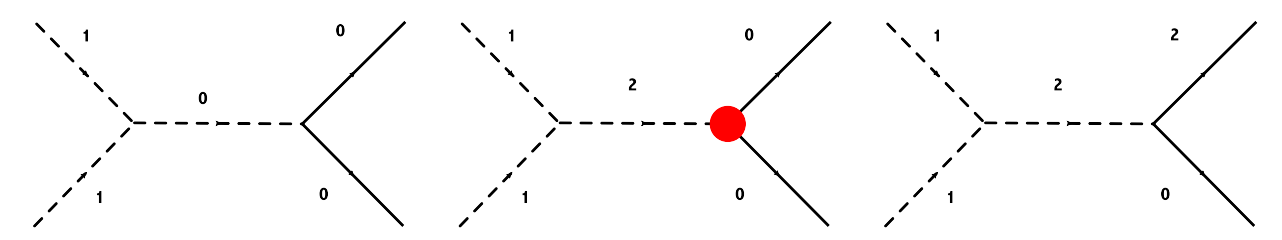

Figure 14. Schematic process included in the L2 scenario. The loop couplings marked as red vertexes.

- L1, where the relic abundance calculation includes only the SM final states (0), i.e. we only consider (co-)annihilation processes $(1)+(1) \rightarrow(0)+(0)$;

- L2, where we allow for the even (2) KK modes in the final state, therefore we also consider the processes $(1)+(1) \rightarrow(2)+(0)$.

In both cases, L1 and L2, the intermediate states are (0) or (2) modes in s-channel or (1) KK modes in t-channel with the couplings at tree level. A schematic depiction of the (co-)annihilation processes can be found in figure 14, where we label with a red dot the loop induced couplings.

In each case, we will also show partial results including:

- L1A and L2A, where we include only annihilation processes mediated by tree level couplings;

- L1AL and L2AL, where only annihilation including loop couplings (s-channel (2) resonances) are considered;

- L1C and L2C, with the full set of co-annihilation processes with tree level couplings only;

- L1CL and L2CL, where co-annihilation including s-channel (2) resonances are included.

For each case, we will study the asymmetric $R_{4} \gg R_{5}$ and symmetric $R_{4}=R_{5}$ cases. Note that the physically meaningful results are given by the most complete case L2CL.

\subsection{L1 scenario}

First we focus on the L1 scenario where the relic abundance calculation includes final states with only a pair SM states (0). In general, an odd (1) state can never decay directly into a pair of SM states but will always decay in a lighter (1) state and an SM particle. Each heavy state produced in (co-)annihilations will therefore undergo a chain decay which will end up with the Dark Matter candidate and SM particles.

\subsubsection{Influence of co-annihilations}

First we examine co-annihilation effects on the relic abundance. As it was stated in section 4 , the mass splittings are very small in our model. We start by adding the next 

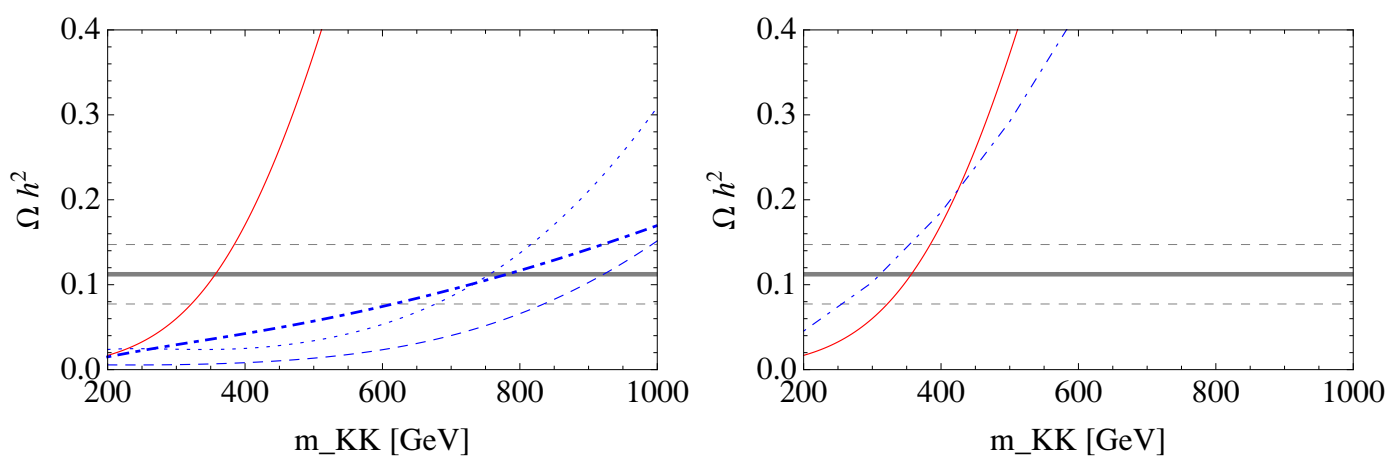

Figure 15. On the left. Relic abundance in the asymmetric L1 scenario including co-annihilation with: right-handed leptons (blue dotted), left-handed leptons (blue dashed), and WZ bosons (blue dot-dashed). On the right. Relic abundance including co-annihilations with all the U(1) and $\mathrm{SU}(2)$-only interacting particles (blue dot-dashed); the red line corresponds to annihilations only.

to LKP particles to co-annihilation channels. In figure 15 we show the influence of coannihilations with: a) right-handed leptons, b) left-handed leptons, and c) electroweak gauge bosons $W^{(1)}$ and $Z^{(1)}$. In all three cases the result is to lower the relic abundance. Right-handed leptons, although having the smallest mass splitting, have weaker interactions than left-handed leptons, which couple also to $\mathrm{SU}(2)$ gauge bosons. Therefore the effective cross section with right-handed leptons will be weaker and the thus the relative reduction in the relic abundance smaller. We should notice that for light $m_{K K} \approx 200 \mathrm{GeV}$ the right-handed leptons induce a small enhancement of the relic abundance, this effect can be explained by very small mass splittings in this region (recall that for $m_{K K} \mathrm{GeV}$ the LKP is the right-handed electron) therefore we add only a small contribution to the cross section while the effective number of degrees of freedom increases, becoming $g_{\text {eff }} \approx 13$ in the $\Lambda=0$ approximation. For the electroweak gauge bosons, although their interactions are of the same strength as those of left-handed leptons, larger mass splittings will reduce the effect of co-annihilations and the relic abundance will be lager than when including much lighter leptons. The result including co-annihilations with all the leptons and $W^{(1)}$ and $Z^{(1)}$ particles is shown in the right panel of figure 15 .

The influence of adding coloured particles is shown in figure 16. They have the largest mass splitting, however the strong SU(3) interactions enhance the effective cross section and highly reduce the relic abundance. We see that the effect is almost independent of the number of new particles we add: the relic abundance does not change whether we add only singlet quarks, both singlet and doublet quarks, or all quarks together with gauge bosons. This means that the main effect is due to the large value of the annihilation cross section of strongly interacting particles and that, once its addition changes the scale of the effective cross section, the result is not sensitive to the number of states added to the model. In the right panel we show the final result of adding all the possible co-annihilation channels. This plot correspond to the most physical scenario at tree level where we include all the co-annihilations. Note however that it is quite difficult to interpret the enhancement of the relic abundance for low $m_{K K}$ masses as compared to the annihilations only as one 
would expect the reduction of $\Omega h^{2}$ for all the range of KK masses. We take therefore the bounds obtained in the case when all the co-annihilations are included as physical bounds $260<m_{K K}<355 \mathrm{GeV}$ in the asymmetric case $R_{4} \gg R_{5}$. On the symmetric orbifold the result is simply twice higher relic abundance as we have two independent Dark Matter candidates with degenerate masses and thus the bounds on the KK mass scale are lowered to $m_{K K}<255 \mathrm{GeV}$.

\subsubsection{Influence of the resonances of (2) modes and loop induced couplings}

We study the influence of adding the loop induced couplings. The formal calculation of the effective couplings was performed in [23]. The mass of the (2) KK particles is close to twice the mass of (1) KK particles. Thus, resonant processes in which the (2) KK modes propagates in the s-channel are important for the calculation of the relic abundance. Such effects in the mUED model were partially investigated in $[26,28,31]$. In the works $[26,28]$ the second KK particle resonances are studied for the LKP annihilation and co-annihilations relevant to the $\mathrm{SU}(2)$ singlet leptons, $e_{S}^{1}$. However, it is found that the second KK resonance processes also play an important role in co-annihilation modes relevant to KK SU(2) doublet leptons and KK Higgs particles. In our model the possible resonances can come from the following particles propagating into $s$-channel: $t_{S / D}^{(2)}, W^{(2)}$, $Z^{(2)}, G^{(2)}, H^{(2)}$. Other (2) states do not directly couple to a pair of SM particles and thus will not contribute in the co-annihilation processes. In table 7 we give the partial widths of the (2) states to a pair of SM particles. For the resonant particles mentioned above the corresponding co-annihilation processes are

$$
\begin{aligned}
& \left.\begin{array}{r}
t_{S / D}^{(1)}+A^{(1)} / Z^{(1)} \\
b^{(1)}+W^{(1)}
\end{array}\right\} \rightarrow t_{S / D}^{(2)} \rightarrow \mathrm{SM} \\
& \left.\begin{array}{c}
A^{(1)}+W^{(1)} \\
l_{D}^{(1)}+\nu^{(1)}
\end{array}\right\} \rightarrow W^{2} \rightarrow \mathrm{SM} \\
& l_{S / D}^{(1)}+\bar{l}_{S / D}^{(1)} \rightarrow Z^{(2)} \rightarrow \mathrm{SM} \\
& \left.\begin{array}{c}
G^{(1)}+G^{(1)} \\
q_{S / D}^{(1)}+\bar{q}_{S / D}^{(1)}
\end{array}\right\} \rightarrow G^{(2)} \rightarrow \mathrm{SM} \\
& \left.\begin{array}{c}
V^{(1)}+V^{(1)} \\
f_{S / D}^{(1)}+\bar{f}_{S / D}^{(1)}
\end{array}\right\} \rightarrow H^{(2)} \rightarrow \mathrm{SM}
\end{aligned}
$$

Note however that the processes can be suppressed by several factors:

1. If the initial particles are heavy, then the process will be Boltzmann suppressed. This condition will be relevant for the initial $G^{(1)}, W^{(1)}, Z^{(1)}, q_{S / D}^{(1)}$ which receive the largest loop corrections to masses.

2. The BR of the (2) to the SM is small. This condition will reduce the resonant contributions but is much less important than the Boltzmann suppression factors. 

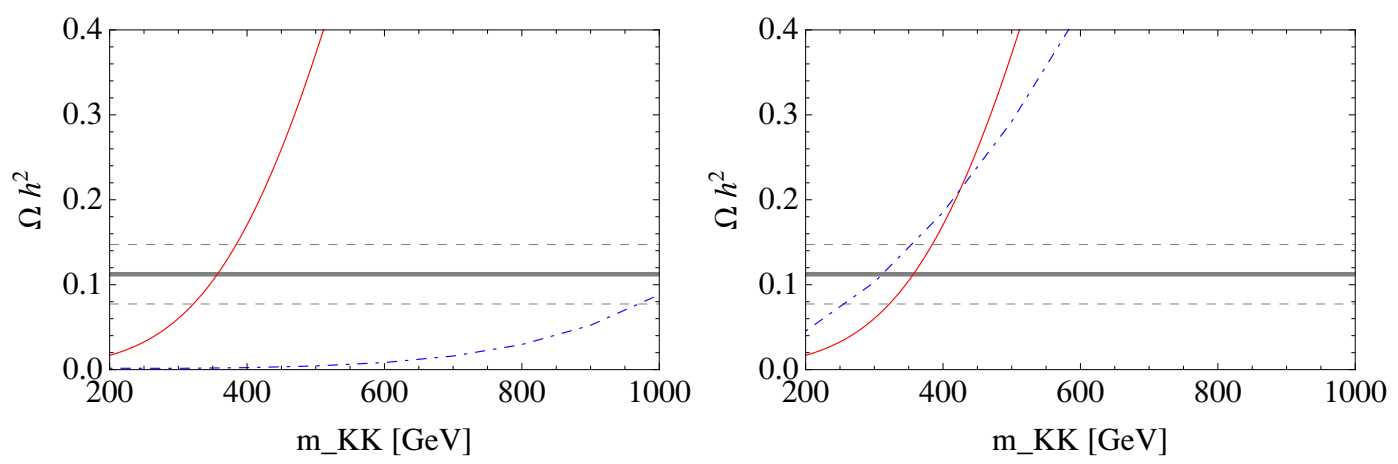

Figure 16. On the left: co-annihilations with all the coloured particles in blue dot dashed. On the right: all the possible co-annihilations are included (blue dot dashed line). The red line corresponds to annihilations only.

\begin{tabular}{|c|c|c|c|}
\hline \multirow[b]{2}{*}{$m_{K K}[\mathrm{GeV}]$} & \multicolumn{3}{|c|}{ Width $[\mathrm{GeV}](\mathrm{Br}[\%])$} \\
\hline & 500 & 1000 & 1500 \\
\hline$Z^{5}$ & $43 \%$ & $45 \%$ & $43 \%$ \\
\hline$W^{5}$ & $43 \%$ & $45 \%$ & $43 \%$ \\
\hline$G^{5}$ & $15 \%$ & $14 \%$ & $14 \%$ \\
\hline$H^{5}$ & $100 \%$ & $100 \%$ & $100 \%$ \\
\hline$A^{5}$ & $100 \%$ & $100 \%$ & $100 \%$ \\
\hline
\end{tabular}

Table 7. Branching ratios of the even (2) KK modes into a SM pair.

3. As the velocities of the particles near the freeze out temperature are non-relativistic, if one is far below the resonance condition, that is the inequality $m_{(1)}+m_{(1)}^{\prime} \ll m_{(2)}$ holds, then the process will be Boltzmann suppressed as well due to large momenta of the incoming particles required to produce the resonance.

We define $m_{K K \text { res }}$ the KK mass at which the resonant condition

$$
m_{(1)}+m_{(1)}^{\prime}=m_{(2)}
$$

is verified. Among many of the kinematically allowed resonances listed above, the contributions of some of them will be highly reduced:

1. $t_{S / D}^{(2)}$ have low branching ratios into SM particles and the initial states producing this resonance are heavy, thus these processes will be both Boltzmann and BR suppressed.

2. $Z^{(2)}$ and $W^{(2)}$ resonances have order 1 BRs to SM particles. The initial states $l^{(1)}$ however are quite light and the process will be Boltzmann suppressed as we are far below the resonance condition.

3. $G^{(2)}$ also has sizeable BRs into SM states. However the (1) modes producing the resonance are the particles with the largest mass splittings in the model, therefore the processes will be strongly suppressed by Boltzmann factors. 

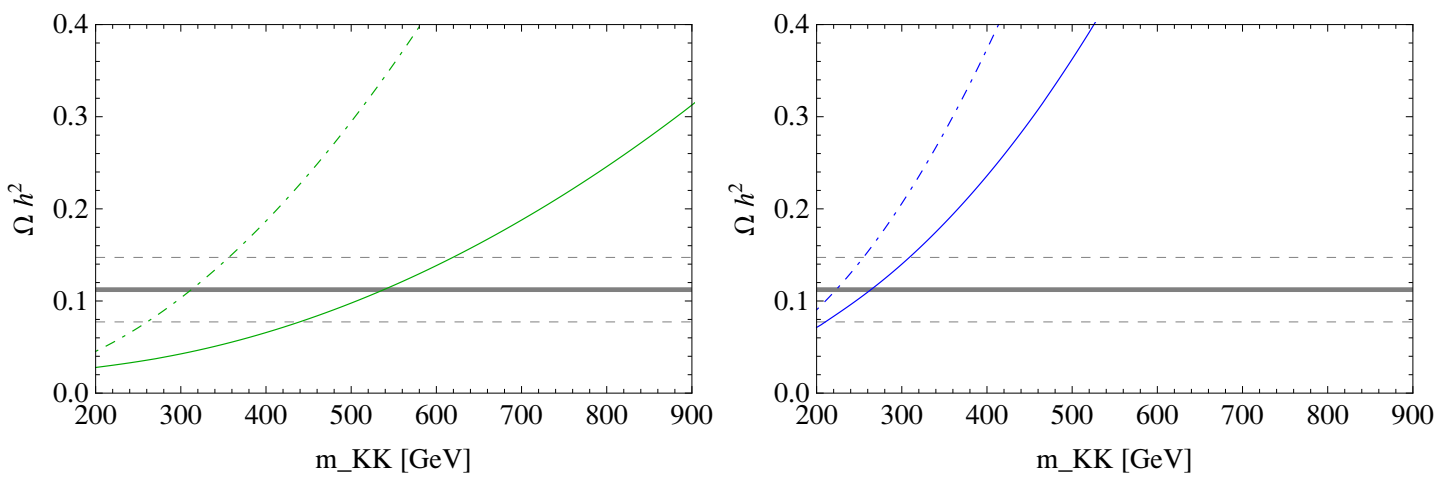

Figure 17. Relic abundance as a function of $m_{K K}$ in the L1 scenario. In both plots we use the convention: dot-dashed lines for co-annihilations (L1C); solid line for co-annihilations + resonances (L1CL). In green (left panel) the asymmetric $R_{4} \gg R_{5}$ case, in blue (right) the symmetric one $R_{4}=R_{5}$.

4. $H^{(2)}$ particles, in spite of very weak couplings are produced by all the (1) coannihilating states including $A^{(1)}$, therefore the effective cross section will be enhanced mainly due to the process $A^{(1)}+A^{(1)} \rightarrow H^{(2)} \rightarrow$ SM. This process will also be very sensitive to the mass of $H^{(2)}$, which is controlled by the free parameter $m_{\text {loc }}$.

The numerical results in the asymmetric (left panel) and symmetric (right panel) cases in L1 scenario are shown in figure 17: we can immediately see that the loop mediated resonances play a crucial role in the relic abundance calculation. In the symmetric case, the effect seems to be smaller: this is due to the fact that the divergent loop corrections to the masses of (2) states are larger, therefore many of the resonances are too heavy to be produced in highly non-relativistic collisions of lighter KK modes.

The effect of including loop induced couplings (tier (2) resonances) when only annihilations are taken into account is quite small. The only resonant particle present in annihilation processes is the Higgs $H^{(2)}$ in the reaction $A^{(1)} A^{(1)} \rightarrow t \bar{t}$. The resonant condition $m_{A^{(1)}}=m_{H^{(2)}}$ is reached from below: for $m_{K K} \leq m_{K K \text { res }}=267 \mathrm{GeV}$ the condition $2 m_{A^{1}}<m_{H^{5}}$ holds therefore we are below the resonance and heavy Higgs can effectively enter as a resonant state lowering the relic abundance. Once the resonant value is exceeded $m_{K K}>m_{K K \text { res }}$ we enter in the regime above the resonance and thus resonant contributions stop. The effect of Higgs resonance can only be seen when only annihilations are taken into account. When we include co-annihilations, all the resonant particles mentioned above contribute. The condition (6.6) can be met for the processes

$$
\begin{aligned}
t_{S / D}^{(1)}+A^{(1)} / Z^{(1)} & \rightarrow t_{S / D}^{(2)} & & \text { at } m_{K K \text { res }} \approx 385 / 680 \mathrm{GeV}, \\
W^{(1)}+A^{(1)} & \rightarrow W^{(2)} & & \text { at } m_{K K \text { res }}=277 \mathrm{GeV}, \\
t_{S / D}^{(1)}+t_{S / D}^{(1)} & \rightarrow G^{(2)} & & \text { at } m_{K K \text { res }}=740 \mathrm{GeV} .
\end{aligned}
$$

In all the above processes, the resonant condition is achieved from above, i.e. for $m_{K K} \leq m_{K K \text { res }}$ we the inequality $2 m_{(1)}>m_{(2)}$ holds and the resonances will be turned on for $m_{K K}$ higher than the threshold values. The result of this is a strong reduction of the 

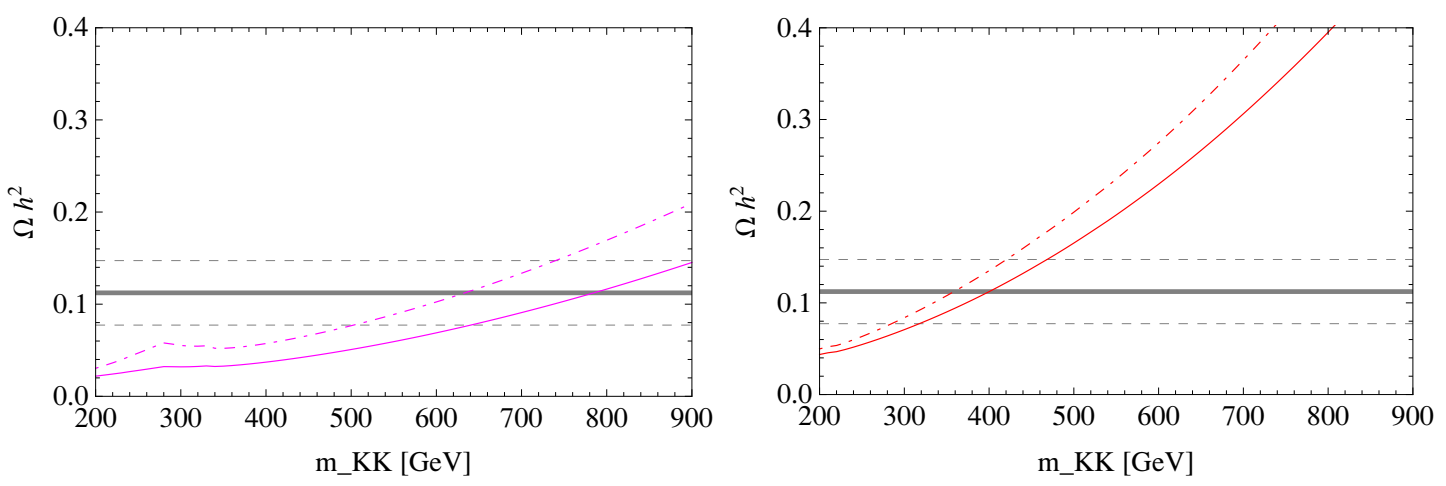

Figure 18. Relic abundance as a function of $m_{K K}$ in the L2 scenario. In all the plots we use the convention: dot-dashed lines for co-annihilations only (L2C), solid line for co-annihilations + resonances (L2CL). In magenta (left panel) the asymmetric case $R_{4} \gg R_{5}$, in red (right panel) the symmetric one $R_{4}=R_{5}$.

relic abundance for high $m_{K K}$ masses (compare dot-dashed and solid lines in figure 17). The co-annihilations into $H^{(2)}$ always pass through the resonant condition from below and contribute at low masses but the minimum corresponding to $A^{(1)} A^{(1)} \rightarrow H^{(2)}$ spread out by stronger contributions of $W^{(2)}$. The main contribution of about $30 \%$ comes although from the $W^{(2)}$ gauge boson as its decay width into SM particle is larger than for other (2) states. The (2) gluons have large decay rates into two SM states but due to their large mass splittings their influence will be strongly suppressed by the Boltzmann factor.

Note finally that here we always fix $m_{\mathrm{loc}}=0 \mathrm{GeV}$, and we will study the dependence of the resonance on this parameter in a following dedicated section.

\subsection{L2 scenario}

In general, an even (2) state can decay into a pair of odd (1) states, into a lighter (2) state plus a SM state or into a pair of SM states. All such final states give comparable partial widths: the first two are mediated by tree level couplings however they are phase space suppressed by the small loop-induced mass splittings (the decays are nearly on-threshold), while the latter is mediated by a loop induced coupling. Each state will therefore undergo a chain decay which will end up with only SM particles if a decay via loop couplings is finally met, or into a pair of Dark Matter candidate if a decay into a pair of odd states is met. In the former case, the annihilation into a (2) state will contribute to the annihilation into SM states. In table 8 we list the inclusive branching ratio into SM states, which takes into account the full decay chains. As we can see, the lighter states (in particular $A^{(2)}$, $H^{(2)}$ and leptons), have a $100 \%$ decay rate into SM final states: this is due to the smallness of the loop corrections that strongly suppresses or closes the phase space of the decays into odd states (the situation for the Higgs may change for large and positive $m_{\text {loc }}^{2}$, which will significantly increase its mass). Therefore, in the following numerical calculations we will consider all the level-(2) modes in the final state as SM states, thus contributing to the (co-)annihilation cross sections. 


\begin{tabular}{|c|cc|}
\hline & \multicolumn{2}{|c|}{ Inclusive BR into SM } \\
$m_{K K}[\mathrm{GeV}]$ & 500 & 1000 \\
\hline$l_{S}^{(2)}$ & $100 \%$ & $100 \%$ \\
$l_{D}^{(2)}$ & $100 \%$ & $100 \%$ \\
$q_{S}^{(2)}$ & $87 \%$ & $86 \%$ \\
$q_{D}^{(2)}$ & $58 \%$ & $54 \%$ \\
$t_{S}^{(2)}$ & $87 \%$ & $80 \%$ \\
$t_{D}^{(2)}$ & $60 \%$ & $52 \%$ \\
$Z^{(2)}$ & $60 \%$ & $60 \%$ \\
$W^{(2)}$ & $60 \%$ & $60 \%$ \\
$G^{(2)}$ & $38 \%$ & $51 \%$ \\
$H^{(2)}$ & $100 \%$ & $100 \%$ \\
$A^{(2)}$ & $100 \%$ & $100 \%$ \\
\hline
\end{tabular}

Table 8. Inclusive Branching Ratios of (2) KK modes into final states with only SM particles.
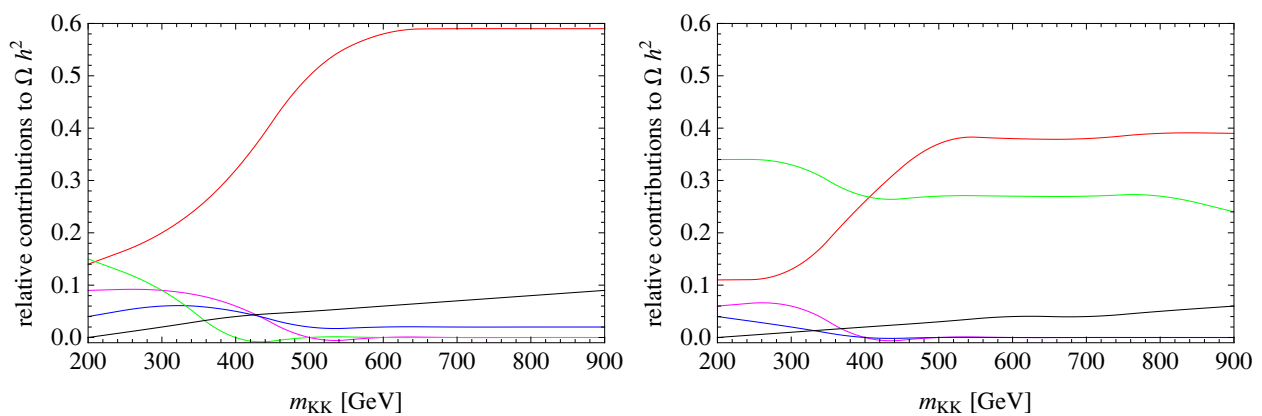

Figure 19. Relative contributions of the partial annihilation cross sections to the relic abundance in L2 scenario when all the co-annihilation channels are open. On the left panel we see the dominant contribution of $l_{S / D}^{(1)} A^{(1)} \rightarrow l A^{(2)}$ in red . Other channel are $l_{D}^{(1)} \nu^{(1)} \rightarrow q \bar{q}$ co-annihilation in green, $l_{D}^{(1)} \nu^{(1)} \rightarrow A H^{(2)}$ in magenta, $q^{(1)} A^{(1)} \rightarrow q A^{(2)}$ in blue and $b_{D}^{(1)} W^{(1)} \rightarrow b W^{(2)}$ in black. On the right panel the same colours are applied for the contributions when we include the loop couplings ((2) resonances). We see the importance of the $W^{(2)}$ resonance in the $l_{D}^{(1)} \nu^{(1)} \rightarrow q \bar{q}$ processes when the loop couplings are turned on.

In the L2 scenario the bounds do not change, compared to L1 case, if we include only annihilations. This is reasonable as the main processes that contribute in both cases are the annihilations $A^{(1)} A^{(1)} \rightarrow W^{+} W^{-}, Z Z, H H$ which are mediated only by tree level couplings. For kinematic reasons, the even (2) states cannot be produced from two $A^{(1)} A^{(1)}$.

Including co-annihilations we have many new final states including $A^{(2)}$ and $H^{(2)}$ as compared to the L1 scenario. Analysis of partial co-annihilation processes present similar behaviour to the L1 scenario therefore here we consider only the final result when all the 


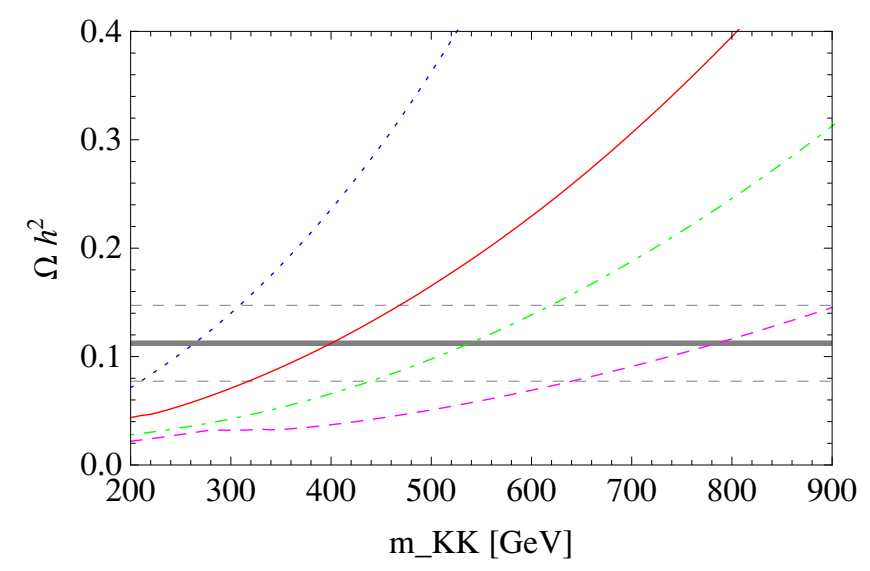

Figure 20. Relic abundance as a function of $m_{K K}$, after taking into account all the co-annihilations and loop couplings. Form top to bottom we have: blue dashed symmetric L1 $R_{4}=R_{5}$, red solid symmetric L2 $R_{4}=R_{5}$, green dot-dashed asymmetric L1 $R_{4} \gg R_{5}$, magenta dashed asymmetric $\mathrm{L} 2 R_{4} \gg R_{5}$.

co-annihilations are taken into account. Due to many new channels including (2) states, as it can be seen form figure 18, the relic abundance bounds on $m_{K K}$ will be pushed up to $m_{K K} \approx 800 \mathrm{GeV}$ in the asymmetric case and to $m_{K K} \approx 400 \mathrm{GeV}$ in the symmetric orbifold. The main contributions are shown in figure 19.

\subsection{Comparison of $m_{K K}$ bounds in L1 and L2 scenarios}

In table 9 we summarise the bounds on the $m_{K K}$ mass scale deduced from the full (including all the co-annihilation channels) relic abundance calculation in the two scenarios L1 and L2 using MicrOMEGAs, while a plot of the relic abundance as a function of $m_{K K}$ is in figure 20. The loop induced couplings do not influence the relic abundance bounds when we open only annihilation channels. The only resonant particle in this case is the heavy Higgs $H^{(2)}$ which produces a resonant minimum but for the $m_{K K}$ far below the expected range compatible with the WMAP data. This behaviour is evident in both scenarios L1 and L2, moreover we have the same bounds for $m_{K K}$ with the expected value set quite low at $360 \mathrm{GeV}$ in the asymmetric orbifolds $R_{4} \gg R_{5}$ and at $290 \mathrm{GeV}$ in the symmetric case $R_{4}=R_{5}$. This estimation gives the first hint of the possible KK scale of extra dimensions but the fully consistent physical result must contain all co-annihilations and loop couplings.

In the L1 scenario when we include all the co-annihilation channels but not loop induced couplings the preferred $m_{K K}$ values lie in the range $260<m_{K K}<355 \mathrm{GeV}$ in the asymmetric case and $m_{K K}<255 \mathrm{GeV}$ in the symmetric orbifold. When we add the loop couplings the resonant contributions lower the $\Omega h^{2}$ value of $52 \%$ and the expected mass scale is pushed up to $440<m_{K K}<620 \mathrm{GeV}$ for $R_{4} \gg R_{5}\left(210<m_{K K}<310 \mathrm{GeV}\right.$ for $\left.R_{4}=R_{5}\right)$. In the L2 scenario, where the (2) states are allowed in the final states, the impact of co-annihilations is more important which translates into higher mass scales of $500<m_{K K}<740 \mathrm{GeV}$ for $R_{4} \gg R_{5}\left(285<m_{K K}<420 \mathrm{GeV}\right.$ for $\left.R_{4}=R_{5}\right)$ correspondingly. Adding loop couplings, the $\Omega h^{2}$ value decrease further of about $36 \%$ (15\%) with 


\begin{tabular}{|c|c|c|}
\hline model & $\begin{array}{c}\text { L1 } \\
m_{K K}[\mathrm{GeV}]\end{array}$ & $\begin{array}{c}\text { L2 } \\
m_{K K}[\mathrm{GeV}]\end{array}$ \\
\hline$R_{4} \gg R_{5}$ & $440-620$ & $640-900$ \\
$R_{4}=R_{5}$ & $210-310$ & $315-470$ \\
\hline
\end{tabular}

Table 9. Preferred ranges for $m_{K K}$ from the relic abundance calculation in the models L1 and L2 for asymmetric radii $R_{4} \gg R_{5}$.

respect to the co-annihilations only setting the mass range at $640<m_{K K}<900 \mathrm{GeV}$ $\left(315<m_{K K}<470 \mathrm{GeV}\right.$ for $\left.R_{4}=R_{5}\right)$.

The relative impact of opening the (2) final states is always to decrease the relic abundance value and in our study we observe a reduction of about $50 \%(45 \%)$ between the L1 and L2 scenarios when all the co-annihilation channels and the loop induced couplings are taken into account. The physical bounds set by the full one loop calculation are summarised in table 9 and the relic abundance as a function of $m_{K K}$ is shown in figure 20 .

\section{Cut-off dependence of the relic abundance}

In this section we are interested in the dependence on the cut-off of the relic abundance calculation. The spectrum of the model as well as the loop induced couplings are logarithmically sensitive to the cut-off of the effective extra dimensional theory. The numerical impact can be seen from table 2, where we show the mass splitting for $m_{K K}=500$ and $800 \mathrm{GeV}$ for two different values of the $\Lambda R$ parameter, and in figure 3 .

The numerical results for the L1 scenario with asymmetric radii can be found in figure 21, and very similar behaviour can be seen for symmetric radii and in L2. When we include only annihilations, the growing cut-off has the effect of increasing the relic abundance (top panels in figure 21). In contrast, when we include all the co-annihilations the situation is reversed - with growing cut off $\Omega h^{2}$ is reduced (bottom panels in figure 21). To understand these effects we need to know first of all which quantities are most influenced by the cut-off. Of course the principal influence will be on the mass spectrum of the particles which is explicitly cut-off dependent. Then, while we include loop induced processes, the effective couplings violating the KK number are also dependent on the cut off scale of the theory.

Let focus first on the two simple cases without resonances due to the loop couplings: L1A (only annihilations) and L1C (all-co-annihilations included), which are shown in the left panels in figure 21. In both cases we do not have any loop induced couplings thus the cut off dependence will only enter in the mass spectrum. With growing cut off, for any given $m_{K K}$, the mass splittings increase (see figure 3). As the annihilations are mediated by the (1) level particles in $t$-channels mainly (for (1) quarks and $A^{(1)}, Z^{(1)}$ ), the larger the mass splittings the smaller the individual cross sections, thus the annihilation cross sections will be suppressed with growing $\Lambda R$. We show the effect in figure 22, where we plot the annihilation cross section $A^{(1)} A^{(1)} \rightarrow S M$ for $m_{K K}=500 \mathrm{GeV}$ as a function of 

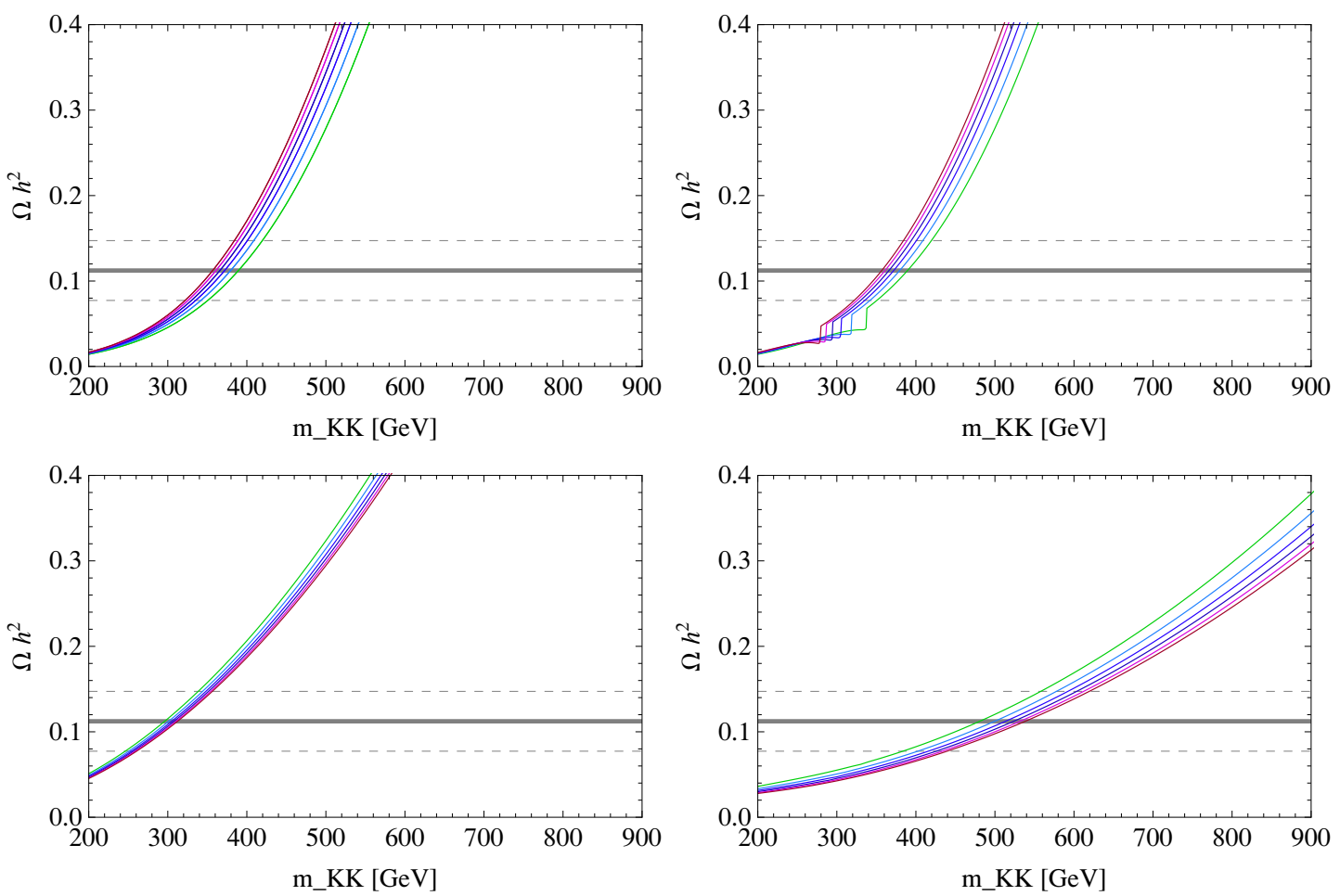

Figure 21. Variations of the relic abundance for $R_{4} \ll R_{5}$ in L1A (top left) and L1AL (top right), L1C (bottom left) and L1CL (bottom right) with the cut-off. Going from magenta to green the cut off is lowered $10>\Lambda R>5$. In black: $\Lambda R=10$ as for every other calculation where we not vary the cut-off.

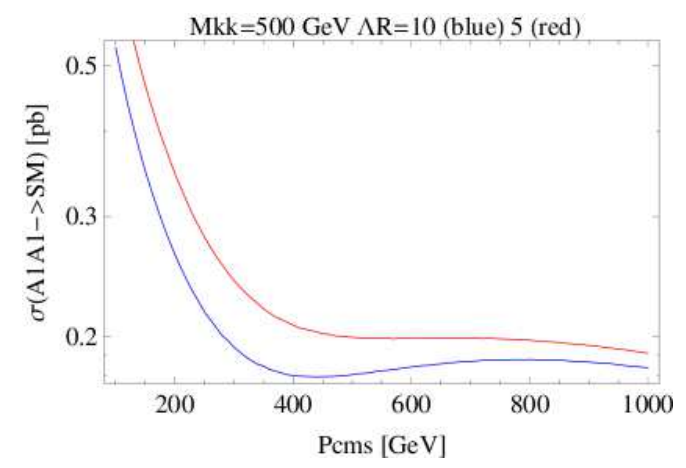

Figure 22. Total annihilation cross section $A^{(1)} A^{(1)} \rightarrow S M$ as a function of $p_{c m s}$ for $M_{K K}=$ $500 \mathrm{GeV}$ and $\Lambda R=5,10$ in red and blue respectively.

the centre of mass momentum $p_{c m s}$. The two lines correspond to $\Lambda R=5$ and 10 , thus showing that the cross section is smaller for larger cut-off. The relic abundance depends on the mean value of $\sigma v$ : we estimate its value by averaging over a Boltzmann distribution of velocities for the DM

$$
f(p) d p \sim p^{2} \exp \left(\frac{p^{2}}{2 m k T}\right) d p
$$




\begin{tabular}{|c|c|c|c|c|c|c|}
\hline & $\Lambda R$ & 2 & 4 & 6 & 8 & 10 \\
\hline L1 & $R_{4} \gg R_{5}$ & $235-380$ & $360-530$ & $405-580$ & $425-605$ & $440-620$ \\
& $R_{4}=R_{5}$ & excluded & $<285$ & $<300$ & $<305$ & $<310$ \\
\hline L2 & $R_{4} \gg R_{5}$ & $500-740$ & $570-820$ & $640-905$ & $675-955$ & $700-990$ \\
& $R_{4}=R_{5}$ & $240-365$ & $295-455$ & $330-480$ & $340-495$ & $350-505$ \\
\hline
\end{tabular}

Table 10. Preferred ranges for $m_{K K}$ (in $\mathrm{GeV}$ ) for different values of $\Lambda R$ in the asymmetric and symmetric radii cases.

where $T=m / x$ with $x=25 .^{3}$ Then, for the mean value $\langle\sigma v\rangle$, we obtain $3.76 \mathrm{pb}$ for $\Lambda R=5$ and $3.20 \mathrm{pb}$ for $\Lambda R=10$, thus confirming that effectively the mean value $\langle\sigma v\rangle$ is larger for larger cut-off scale. This would imply that the relic abundance is larger for larger cut-off, as confirmed in the annihilation-only case L1A.

The case with co-annihilation shows an exactly opposite behaviour. However, a larger cut-off would imply that the individual co-annihilation cross sections decrease; furthermore, the larger mass splittings will also suppress the contribution of the co-annihilation channels. We might then conclude that the larger the cut-off the larger the relic abundance, similarly to the annihilation only case. However, increased mass splittings have another effect: the effective number of degrees of freedom $g_{\text {eff }}$ is also reduced. The mass splitting increases more importantly for the leptons, while gauge bosons $W^{(1)}$ and $Z^{(1)}$ also receive a cutoff independent contribution from the $W$ and $Z$ mass. As leptons contribute more to the degrees of freedom than to the average cross section, when increasing the cut-off the decrease in the degrees of freedom dominates and the relic abundance is reduced, as we observe in the numerical results.

Including the loop couplings does not change the trend, as it can be seen in the right panels in figure 21. The only visible effect is a change in the features due to the resonant exchange of tier (2) states, namely $H^{(2)}$ and $A^{(2)}$ due to the change in the resonant value of $m_{K K}$.

We summarise the numerical bounds for different values of the cut-off $\Lambda R$ for L1 and for the complete model L2 in table 10. Note that the allowed mass values vary from about $200 \mathrm{GeV}$ for the symmetric case - a value that is already excluded by the accelerator searches, up to $1 \mathrm{TeV}$ - a region which is viable as not yet excluded by the LHC data.

\section{Localised Higgs mass dependence}

In this section we vary the $m_{\text {loc }}$ parameter, which is a free parameter of the model. It corresponds to the Higgs mass operator localised on the singular points of the orbifold.

As it was mentioned in section 4 , the main processes mediated by the resonant $H^{(2)}$ are from the $A^{(1)} A^{(1)}$ states. The resonant condition in eq. (6.6) depends on the loop

\footnotetext{
${ }^{3}$ We can safely assume $x$ constant. We have checked that during the relic abundance calculation indeed its value varies within the range of $22-26$ for all the relevant $m_{K K}$ and for all the assumed values of $\Lambda R$.
} 


\begin{tabular}{|c|c|c|}
\hline$m_{\text {loc }}$ & $m_{K K \text { res }}[\mathrm{GeV}]$ & $m_{K K}>[\mathrm{GeV}]$ \\
\hline 0 & 267 & 0 \\
100 & 353 & 351 \\
200 & 544 & 558 \\
300 & 769 & 731 \\
400 & 1005 & 886 \\
500 & 1248 & 1028 \\
\hline
\end{tabular}

Table 11. Values of $m_{K K \text { res }}$ corresponding to resonance $2 m_{A^{(1)}}=m_{H^{(2)}}$ and the lower bounds for $m_{K K}$ obtained from the electroweak precision constraints (in the asymmetric case) for different values of $m_{\mathrm{loc}}$.

corrections to the $A^{(1)}$ and $H^{(2)}$ masses. If we parameterise

$$
m_{A^{(1)}}^{2}=m_{K K}^{2}\left(1+\delta_{A}\right), \quad m_{H^{(2)}}^{2}=4 m_{K K}^{2}\left(1+\delta_{H}\right)+m_{H}^{2}+m_{\mathrm{loc}}^{2}
$$

the resonant condition reads

$$
m_{K K}^{2} \leq \frac{m_{h}^{2}+m_{\mathrm{loc}}^{2}}{4 \delta_{A}-\delta_{H}}
$$

Numerically, it turns out that $\delta_{A} \sim 0$ and $\delta_{H}<0$, therefore a resonance is possible as long as $m_{\mathrm{loc}}^{2}>-m_{H}^{2}$. In the following we will focus on positive values of the localised mass square, which will give rise to resonance for large values of $m_{K K}$. In table 11 we show a list of the resonant $m_{K K \text { res }}$ and lower bound on $m_{K K}$ for various values of $m_{\text {loc }}$ : we find that for $110 \mathrm{GeV}<m_{\text {loc }}<236 \mathrm{GeV}$, the resonance always appears below the upper bound on $m_{K K}$.

Finally we study the influence of the resonant $H^{(2)}$ exchange on the relic abundance. First we focus on the L1A case, where the effect is more visible: the only relevant annihilation process is $A^{(1)} A^{(1)} \rightarrow t \bar{t}$. In figure 23 we show the relic abundance as a function of $m_{K K}$ for various values of $m_{\mathrm{loc}}$. For $m_{\mathrm{loc}}=0 \mathrm{GeV}$, the resonance produces a dip at low $m_{K K}$ where the relic abundance is well below the WMAP preferred region.

With increasing $m_{\text {loc }}$, the resonant dip start becoming relevant opening up extra parameter space: for instance, for $m_{\text {loc }}=300 \mathrm{GeV}$, we observe that a small region $760<$ $m_{K K}<780 \mathrm{GeV}$ also gives the WMAP relic abundance together with the standard low $m_{K K}$ region $440<m_{K K}<620 \mathrm{GeV}$. After the resonant condition is met, which gives the position of the local minimum for the relic abundance, the resonance becomes rapidly ineffective as the DM states have a mass above the KK Higgs one. Note that the increase in the depth of the peak for increasing $m_{\text {loc }}$ is not sufficient and for large values of the localised mass the dip will not be able to touch the WMAP preferred region and no extra parameter space opens up. In the L1 model, this happens for $m_{\mathrm{loc}}>400 \mathrm{GeV}$.

These features remain also in the complete model, depicted in the right panel of the figure 23, however we see that the impact of the resonance is greatly suppressed. This is due to the fact that the only relevant resonant process remains $A^{(1)} A^{(1)} \rightarrow H^{(2)} \rightarrow t \bar{t}$, which is however diluted by the full set of co-annihilation processes. 

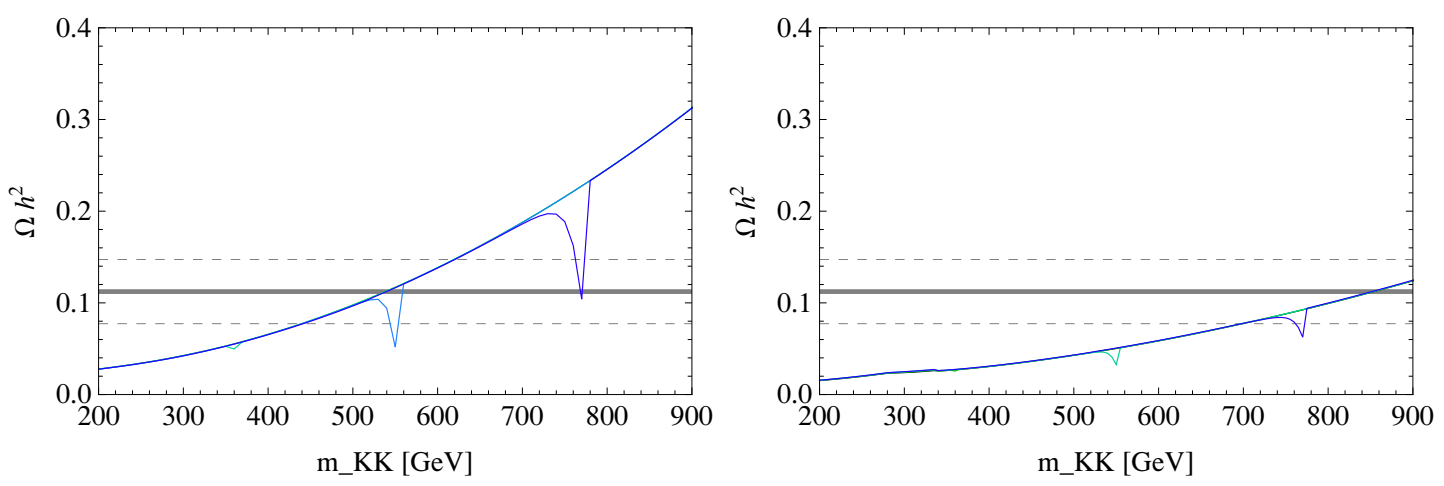

Figure 23. Relic abundance as a function of $m_{K K}$ with all co-annihilations and resonances included. We are particularly interested in the $H^{(2)}$ resonance as a function of $m_{\mathrm{loc}}$. In the plots the dips correspond to the resonances of $H^{(2)}$ at, from left to right, $m_{\mathrm{loc}}=0,100,200,300 \mathrm{GeV}$. The first two minima are almost invisible, the large two dips correspond to $m_{\mathrm{loc}}=200,300 \mathrm{GeV}$. On the left: asymmetric L1CL. On the right: asymmetric L2CL.
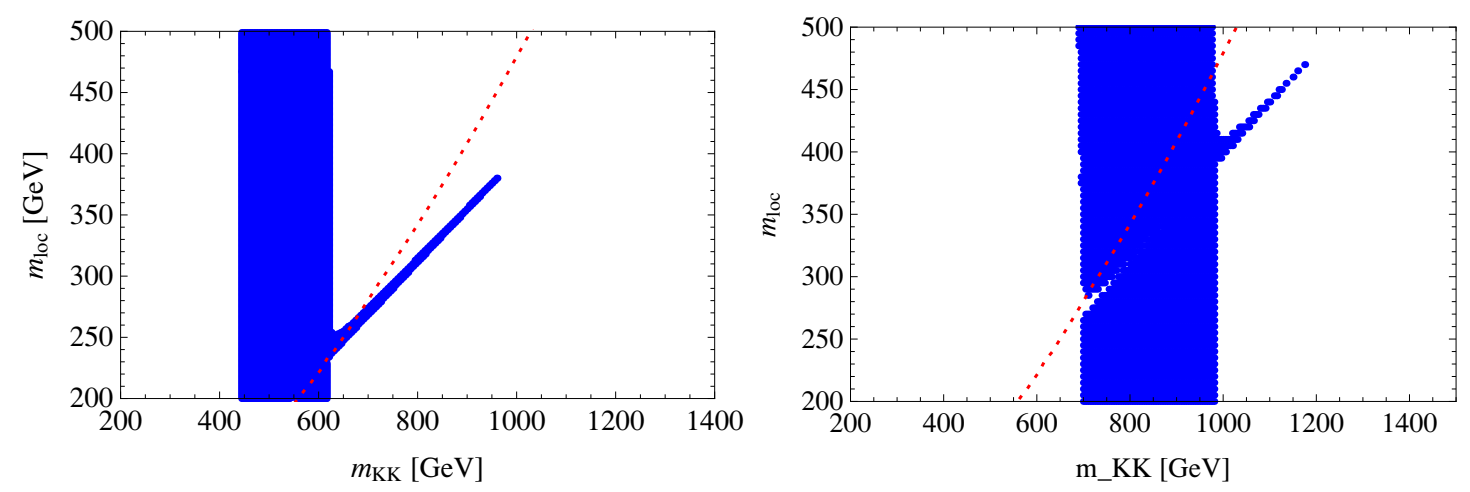

Figure 24. We show in blue the region of $m_{K K}-m_{\text {loc }}$ preferred by WMAP in blue. The parameter space above the red line is disfavoured by the $\rho$ parameter. In the left panel: asymmetric L1 model. In the right panel: asymmetric L2 scenario.

To be complete we scan the parameter space $m_{K K}-m_{\text {loc }}$. The resonance has an important effect on the WMAP preferred parameter range, as it is shown in the right panel of figure 24. While the usual region $640<m_{K K}<900 \mathrm{GeV}$ is still open, for $m_{\text {loc }}>$ $400 \mathrm{GeV}$ a funnel region opens up where the resonant $H^{(2)}$ exchange dominates. Such region corresponds approximately to the resonant condition in eq. (8.2), giving an allowed region around $m_{K K} \sim 2.5 m_{\text {loc }}$. Note that the funnel region closes up for $m_{\text {loc }}>470 \mathrm{GeV}$ (corresponding to $m_{K K} \sim 1200 \mathrm{GeV}$ ), where the dip in the relic abundance is not deep enough to touch the preferred WMAP region. Interestingly, this implies that even the resonant funnel region admits an upper bound on $m_{K K}$. The bound from the $\rho$ parameter, indicated by a red line in figure 24 , shows that large values of $m_{\text {loc }}$ are disfavoured, however the funnel region is still in the allowed parameter space. A similar behaviour occurs in the L1 model, depicted in the left panel of figure 24 . 

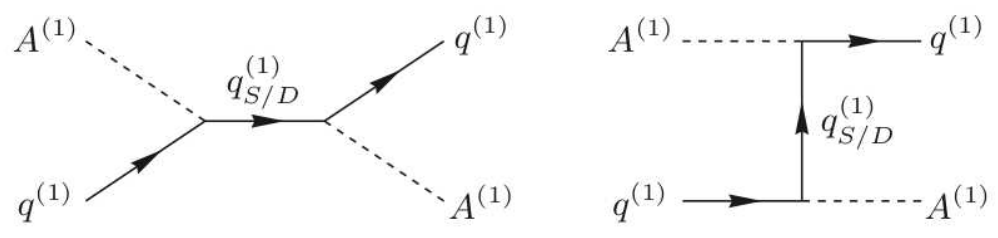

Figure 25. Feynman diagrams for direct detection $A^{(1)} q \rightarrow A^{(1)} q$ via $q_{S / D}^{(1)}$ in $s$ and $t$ channel; $q$ stands for the quarks present in the nucleon, thus mainly up and down.

\section{Direct detection bounds}

A number of experiments are currently searching for a Cold Dark Matter. Their sensitivity is being continuously improved and the upper limits are upgraded regularly. The best upper limit for the WIMP-proton spin independent cross section has been recently obtained by Xenon, $\sigma_{p \chi}^{S I}<3.4 \times 10^{-8} \mathrm{pb}$ for a $55 \mathrm{GeV}$ WIMP and CDMS $\sigma_{p \chi}^{S I}<3.8 \times 10^{-8} \mathrm{pb}$ for a $70 \mathrm{GeV}$ WIMP.

In the extra-dimensional models the potential dark matter candidate is usually a KK gauge boson, but it is also possible that a KK scalar, a KK graviton or a KK neutrino is the Lightest KK particle. In the minimal UED model (mUED) the dark matter candidate is the $\mathrm{U}(1)$ gauge boson $B^{1} .{ }^{4}$ In our six-dimensional model the dark matter candidate is the first level photon $A^{(1)}$ which is a scalar particle. Thus its interaction properties will be different from the 5D UED models where the first level photon is a vector particle, as spindependent interactions are absent. Therefore the direct detection will be mediated only by spin-independent interactions. The case where the direct detection signal is mediated only by the SI interaction is much less discriminating with respect to which model is being detected. The only information that can be used is the total cross section and the mass of the DM particle.

We compute the direct detection signal using MicrOMEGAs v2.4.1. The input model is as defined in previous subsection. The direct detection signal is mediated by the processes showed in figure 25. The interactions of $A^{(1)}$ with all the SM quark are mediated by the level one quarks $q_{D / S}^{(1)}$ in $s$ and $t$ channel. Interactions with heavy quarks $c, b, t$ are also mediated by the SM Higgs boson $h$ in $t$-channel. The Yukawa couplings with light quarks can be safely neglected as they are proportional to the mass of the quark.

The model has two free parameters: $m_{\mathrm{loc}}$ and $\Lambda R$. The latter only influences the KK Higgs masses and it can only enter via a $H^{(2)} t$-channel exchange, which is negligible as it is suppressed by one loop compared to the tree level diagrams in figure 25 . On the other hand, the result depends crucially on the cut-off $\Lambda R$ : in fact, increasing the cut-off will increase the mass of the tier (1) quarks and thus suppress the scattering. The effect is important due to the closeness between the mass of the DM candidate $A^{(1)}$ and the heavy

\footnotetext{
${ }^{4}$ More precisely the Dark Matter Candidate is the level 1 photon $A^{(1)}$ - a linear superposition of $B^{(1)}$ and $W_{3}^{(1)}$ — but the mixing angle can be safely neglected.
} 
quarks. In our numerical study, we vary the $\Lambda R$ parameter in the range

$$
2<\Lambda R<10,
$$

The results are shown in figure 26 where we plot the spin-independent cross section as a function of $m_{K K}$ for five choices of $\Lambda R$ (in blue) as well as various bounds from experiences: Zepelin [32], Edelweiss + CDMS combined data [33], Xenon limits from 2011 [34] and 2012 [35] as well as projection limits in Xenon for 2017 extracted from DMTools. The numerical values for $\Lambda R=2,4,6,8$, and 10 are given in table 12. The best bound is coming from Xenon 2011, and it ranges from $m_{K K}>520$ to $760 \mathrm{GeV}$, increasing for smaller cut-offs. The bound is also independent on the radii, being the same foe asymmetric and symmetric ones. It is interesting to compare the bounds in table 12 with the preferred WMAP ranges in table 10: we immediately see that the WMAP preferred ranges move to higher values of $m_{K K}$ for larger cut-offs, while the bounds from direct detection decrease. Numerically, the symmetric case seems to be completely excluded, while low cut-off values $\Lambda R \lesssim 3$ are excluded for asymmetric radii. This is clear from figure 27 , where we show in yellow the region preferred by WMPA in the $\Lambda R-m_{K K}$ region together with the bounds from direct detection experiments: the best bound is given by Xenon100 (solid blue line). It is also interesting to compare the Direct Detection bound with bounds from accelerators (LHC): the strongest bound should come from dilepton resonances from the decays of the even tiers (2). An estimate [22] shows that the bound, after the analysis of the 2011 data at $7 \mathrm{TeV}$, is $m_{K K}<575 \mathrm{GeV}$ in the asymmetric case, and $m_{K K}<440 \mathrm{GeV}$ in the symmetric one. ${ }^{5}$ The bounds have been computed for $\Lambda R=10$, however they should bear a minor dependence on its precise value and this be near constant in the region under investigation. Direct detection bounds are therefore competitive with collider ones, and in particular dominate for smaller mass splitting (small $\Lambda R$ ) and in the symmetric case.

One way out of this conclusion is the funnel region opened by the $H^{(2)}$ resonance, which allows for large $m_{K K}$. The strong direct detection bounds can also be compared to the bounds on other UED models in 2 dimensions, like the chiral square, where smaller cross sections are obtained [38].

\section{Conclusion}

We have studied the dark matter relic abundance on the Universal Real Projective Plane model. This model is an extra dimensional model with two extra dimensions compactified on a twisted bottle, or real projective plane. This peculiar geometry brings an extra argument for the stability of dark matter, which is not an imposed parity but just a remnant of the 6-dimensional Lorentz invariance partially broken by the compactification. One of the main features of this model is the highly degenerate spectrum in each KaluzaKlein tier, due to the smaller loop corrections compared to other UED models. This in turn implies that co-annihilation effects and higher modes are important in the calculation of the relic abundance. We have performed a detailed analytic and numerical study which

\footnotetext{
${ }^{5}$ Indirect bounds on $m_{K K}$ can be extracted from the Higgs physics [36, 37], and may be important.
} 


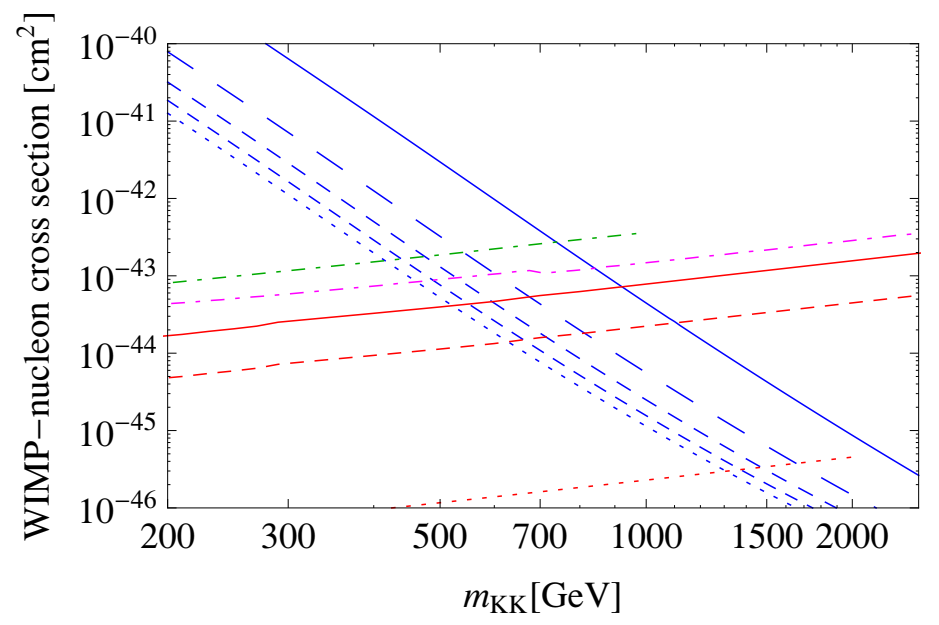

Figure 26. Direct detection bounds on $m_{K K}$ for the $\mathrm{RP}^{2}$ for $\Lambda R=2,4,6,8,10$. The WIMP proton cross sections in blue correspond to the 5 values of the cut-off: from blue solid line $(\Lambda R=2)$ down to blue dotted line $(\Lambda R=10)$. In red we show the Xenon limit: solid - 2011, dashed 2012, dotted - 2017. In dot-dashed green/magenta the limits from Zepelin/CDMS+EDELWEISS.

\begin{tabular}{|c||c|c|c|c|c||c|}
\hline Experiment & Zepelin & $\begin{array}{c}\text { Edelweiss } \\
+ \text { CDMS }\end{array}$ & Xenon2011 & Xenon2012 & Xenon2017 & $\Lambda R$ \\
\hline $10^{-44} \sigma[\mathrm{cm}]^{2}$ & 27.089 & 12.589 & 6.422 & 2.061 & 0.05 & 2 \\
$m_{K K}[\mathrm{GeV}]$ & 740 & 840 & 940 & 1140 & 2200 & \\
\hline $10^{-44} \sigma[\mathrm{cm}]^{2}$ & 185 & 97.285 & 45.308 & 14.555 & 0.251 & 4 \\
$m_{K K}[\mathrm{GeV}]$ & 540 & 600 & 680 & 820 & 1650 & \\
\hline $10^{-44} \sigma[\mathrm{cm}]^{2}$ & 435.537 & 209.142 & 97.285 & 32.004 & 0.498 & 6 \\
$m_{K K}[\mathrm{GeV}]$ & 470 & 530 & 600 & 720 & 1460 & \\
\hline $10^{-44} \sigma[\mathrm{cm}]^{2}$ & 643.533 & 337.704 & 166.731 & 54.356 & 0.685 & 8 \\
$m_{K K}[\mathrm{GeV}]$ & 440 & 490 & 550 & 660 & 1380 & \\
\hline $10^{-44} \sigma[\mathrm{cm}]^{2}$ & 14.718 & 7.615 & 4.165 & 1.38922 & 0.028 & 10 \\
$m_{K K}[\mathrm{GeV}]$ & 415 & 465 & 520 & 630 & 1315 & \\
\hline
\end{tabular}

Table 12. Upper bounds on $m_{K K}$ mass from different direct detection experiments.

allows to obtain a range of KK-masses in which the model is consistent with the relic abundance by providing a good candidate for the stable dark matter particle. Our results can be compared to calculations performed for other UED models [28, 31, 39, 40].

Our complete computation of the relic density of dark matter in the Universal Real Projective Plane model includes all effects of tier (1) and tier (2) states together with the precise spectra calculated at one-loop level, thus allows to understand in detail the role of 

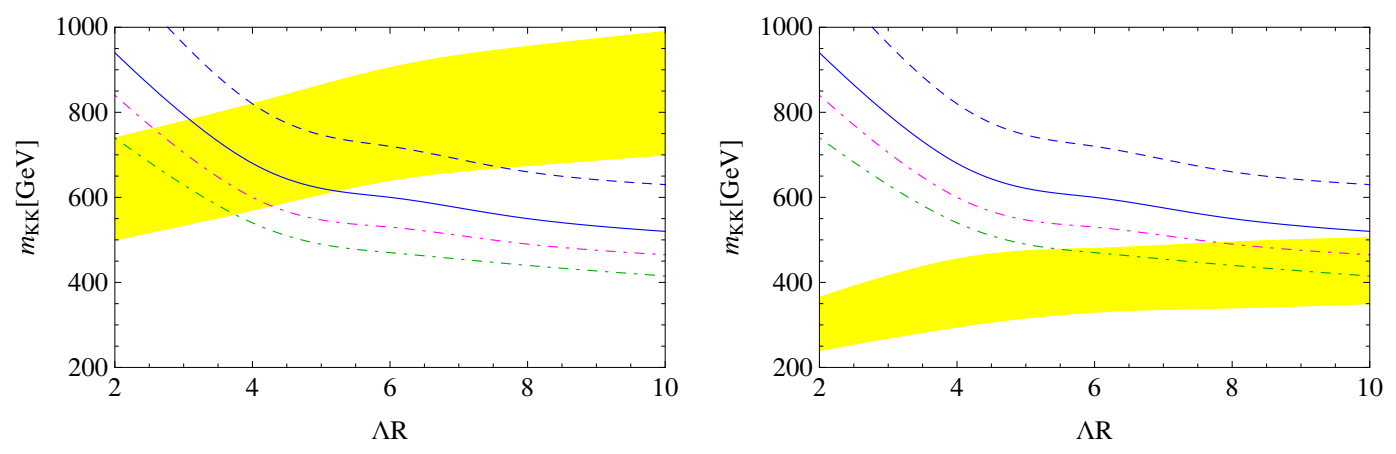

Figure 27. In yellow we show the WMAP preferred region for $m_{K K}$ as a function of the cut-off scale $\Lambda R$ for asymmetric (left) and symmetric (right) radii. The lines delimit the lower bounds from direct detection experiments: Zepelin (dot-dashed green), CDMS+EDELWEISS (green dotdashed), Xenon 2011 (blue solid), Xenon 2012 projection (blue dashed), Xenon 2017 projection (blue dotted). The allowed $m_{K K}$ regions are above the direct detection lines.

the different ingredients active in this model: annihilation versus co-annihilation, different particles contributions for DM observables, effect of the cut-off and of localised Higgs mass terms, perspective of direct versus indirect bounds, implication for the LHC searches. We find that the preferred range for $m_{K K}$ is $700<m_{K K}<990 \mathrm{GeV}$ for the asymmetric case and $350<m_{K K}<505 \mathrm{GeV}$ for degenerate radii, in both cases for maximal cut-off $\Lambda R=10$. Decreasing the cut-off, the preferred ranges are reduced. Such ranges are well into the reach of the LHC. We also find that the tier (2) Higgs resonance open a funnel region in the parameter space that allows to increase $m_{K K}$ up to $\sim 1200 \mathrm{GeV}$. We also computed the SI direct detection cross section for the scalar Dark matter candidate, and we found values close to the experimental bounds: in fact, we found that the 2011 Xenon100 results already exclude the degenerate radii case, and severely constraints the low cut-off case for asymmetric radii. We expect that by the end of 2017, Xenon may be able to exclude the full parameter space. Direct detection bounds are also competitive with LHC bounds, even though a complete study of the Universal $\mathrm{RP}^{2}$ at the hadron collider has not been completed.

Dark Matter in extra dimensions is still a very plausible candidate to explain the matter density in the Universe, and it offers the possibility of an exact symmetry deriving from the properties of the compact space. Furthermore, the mass scales required by the WMAP data are in the range presently being explored at the LHC. We explored a particular realisation of this idea on a twisted bottle, while many other spaces and topologies are still unexplored.

\section{Acknowledgments}

We would like to thank A. Pukhov for his help with MicrOMEGAs. A.A. acknowledges partial support from the European Union FP7 ITN INVISIBLES (Marie Curie Actions, PITN-GA-2011-289442). G.C. acknowledge the hospitality of King's College London during the completion of this work. 
Open Access. This article is distributed under the terms of the Creative Commons Attribution License which permits any use, distribution and reproduction in any medium, provided the original author(s) and source are credited.

\section{References}

[1] G. Servant and T.M. Tait, Is the lightest Kaluza-Klein particle a viable dark matter candidate?, Nucl. Phys. B 650 (2003) 391 [hep-ph/0206071] [INSPIRE].

[2] G. Cacciapaglia, A. Deandrea and J. Llodra-Perez, A Dark matter candidate from Lorentz invariance in 6D, JHEP 03 (2010) 083 [arXiv:0907.4993] [INSPIRE].

[3] P. Gondolo and G. Gelmini, Cosmic abundances of stable particles: Improved analysis, Nucl. Phys. B 360 (1991) 145 [inSPIRE].

[4] J. Edsjö and P. Gondolo, Neutralino relic density including coannihilations, Phys. Rev. D 56 (1997) 1879 [hep-ph/9704361] [InSPIRE].

[5] G. Bélanger, F. Boudjema, A. Pukhov and A. Semenov, MicrOMEGAs: A Program for calculating the relic density in the MSSM, Comput. Phys. Commun. 149 (2002) 103 [hep-ph/0112278] [INSPIRE].

[6] G. Bélanger, F. Boudjema, A. Pukhov and A. Semenov, MicrOMEGAs: Version 1.3, Comput. Phys. Commun. 174 (2006) 577 [hep-ph/0405253] [INSPIRE].

[7] WMAP collaboration, E. Komatsu et al., Seven-Year Wilkinson Microwave Anisotropy Probe (WMAP) Observations: Cosmological Interpretation, Astrophys. J. Suppl. 192 (2011) 18 [arXiv:1001.4538] [INSPIRE].

[8] M. Kamionkowski and M.S. Turner, Thermal relics: do we know their abundances?, Phys. Rev. D 42 (1990) 3310 [INSPIRE].

[9] P. Salati, Quintessence and the relic density of neutralinos, Phys. Lett. B 571 (2003) 121 [astro-ph/0207396] [INSPIRE].

[10] S. Profumo and P. Ullio, SUSY dark matter and quintessence, JCAP 11 (2003) 006 [hep-ph/0309220] [INSPIRE].

[11] D.J. Chung, L.L. Everett, K. Kong and K.T. Matchev, Connecting LHC, ILC and Quintessence, JHEP 10 (2007) 016 [arXiv:0706.2375] [INSPIRE].

[12] A. Arbey and F. Mahmoudi, SUSY constraints from relic density: High sensitivity to pre-BBN expansion rate, Phys. Lett. B 669 (2008) 46 [arXiv:0803.0741] [INSPIRE].

[13] T. Moroi and L. Randall, Wino cold dark matter from anomaly mediated SUSY breaking, Nucl. Phys. B 570 (2000) 455 [hep-ph/9906527] [INSPIRE].

[14] G.F. Giudice, E.W. Kolb and A. Riotto, Largest temperature of the radiation era and its cosmological implications, Phys. Rev. D 64 (2001) 023508 [hep-ph/0005123] [INSPIRE].

[15] N. Fornengo, A. Riotto and S. Scopel, Supersymmetric dark matter and the reheating temperature of the universe, Phys. Rev. D 67 (2003) 023514 [hep-ph/0208072] [INSPIRE].

[16] G. Gelmini, P. Gondolo, A. Soldatenko and C.E. Yaguna, The effect of a late decaying scalar on the neutralino relic density, Phys. Rev. D 74 (2006) 083514 [hep-ph/0605016] [INSPIRE].

[17] A. Arbey and F. Mahmoudi, SUSY constraints, relic density and very early Universe, JHEP 05 (2010) 051 [arXiv:0906.0368] [INSPIRE]. 
[18] A. Arbey and F. Mahmoudi, SuperIso Relic: A Program for calculating relic density and flavor physics observables in Supersymmetry, Comput. Phys. Commun. 181 (2010) 1277 [arXiv: 0906.0369] [INSPIRE].

[19] A. Arbey and F. Mahmoudi, SuperIso Relic v3.0: A program for calculating relic density and flavour physics observables: Extension to NMSSM, Comput. Phys. Commun. 182 (2011) 1582 [inSPIRE].

[20] A. Arbey, AlterBBN: A program for calculating the BBN abundances of the elements in alternative cosmologies, Comput. Phys. Commun. 183 (2012) 1822 [arXiv:1106.1363] [INSPIRE].

[21] H. Dohi and K.-y. Oda, Universal extra dimensions on real projective plane, Phys. Lett. B 692 (2010) 114 [arXiv:1004.3722] [INSPIRE].

[22] G. Cacciapaglia and B. Kubik, Even tiers and resonances on the Real Projective Plane, arXiv: 1209.6556 [INSPIRE].

[23] G. Cacciapaglia, A. Deandrea and J. Llodra-Perez, The Universal Real Projective Plane: LHC phenomenology at one Loop, JHEP 10 (2011) 146 [arXiv:1104.3800] [INSPIRE].

[24] B.A. Dobrescu and E. Ponton, Chiral compactification on a square, JHEP 03 (2004) 071 [hep-th/0401032] [INSPIRE].

[25] G. Burdman, B.A. Dobrescu and E. Ponton, Six-dimensional gauge theory on the chiral square, JHEP 02 (2006) 033 [hep-ph/0506334] [INSPIRE].

[26] M. Kakizaki, S. Matsumoto, Y. Sato and M. Senami, Relic abundance of LKP dark matter in UED model including effects of second KK resonances, Nucl. Phys. B 735 (2006) 84 [hep-ph/0508283] [INSPIRE].

[27] T. Appelquist, H.-C. Cheng and B.A. Dobrescu, Bounds on universal extra dimensions, Phys. Rev. D 64 (2001) 035002 [hep-ph/0012100] [InSPIRE].

[28] M. Kakizaki, S. Matsumoto and M. Senami, Relic abundance of dark matter in the minimal universal extra dimension model, Phys. Rev. D 74 (2006) 023504 [hep-ph/0605280] [INSPIRE].

[29] N.D. Christensen and C. Duhr, FeynRules - Feynman rules made easy, Comput. Phys. Commun. 180 (2009) 1614 [arXiv:0806.4194] [InSPIRE].

[30] A. Pukhov, CalcHEP 2.3: MSSM, structure functions, event generation, batchs and generation of matrix elements for other packages, hep-ph/0412191 [INSPIRE].

[31] G. Bélanger, M. Kakizaki and A. Pukhov, Dark matter in UED: the role of the second KK level, JCAP 02 (2011) 009 [arXiv: 1012.2577] [INSPIRE].

[32] D.Y. Akimov et al., WIMP-nucleon cross-section results from the second science run of ZEPLIN-III, Phys. Lett. B 709 (2012) 14 [arXiv:1110.4769] [InSPIRE].

[33] CDMS and EDELWEISS collaborations, Z. Ahmed et al., Combined Limits on WIMPs from the CDMS and EDELWEISS Experiments, Phys. Rev. D 84 (2011) 011102 [arXiv:1105.3377] [INSPIRE].

[34] XENON100 collaboration, E. Aprile et al., Dark Matter Results from 100 Live Days of XENON100 Data, Phys. Rev. Lett. 107 (2011) 131302 [arXiv:1104.2549] [INSPIRE].

[35] XENON100 collaboration, E. Aprile et al., The XENON100 Dark Matter Experiment, Astropart. Phys. 35 (2012) 573 [arXiv:1107.2155] [INSPIRE]. 
[36] K. Nishiwaki, K.-y. Oda, N. Okuda and R. Watanabe, A Bound on Universal Extra Dimension Models from up to 2 fb ${ }^{-1}$ of LHC Data at 7 TeV, Phys. Lett. B 707 (2012) 506 [arXiv:1108.1764] [INSPIRE].

[37] T. Kakuda, K. Nishiwaki, K.-y. Oda, N. Okuda and R. Watanabe, Higgs at ILC in Universal Extra Dimensions in Light of Recent LHC Data, arXiv:1202.6231 [INSPIRE].

[38] B.A. Dobrescu, D. Hooper, K. Kong and R. Mahbubani, Spinless photon dark matter from two universal extra dimensions, JCAP 10 (2007) 012 [arXiv:0706.3409] [INSPIRE].

[39] K. Kong and K.T. Matchev, Precise calculation of the relic density of Kaluza-Klein dark matter in universal extra dimensions, JHEP 01 (2006) 038 [hep-ph/0509119] [INSPIRE].

[40] F. Burnell and G.D. Kribs, The abundance of Kaluza-Klein dark matter with coannihilation, Phys. Rev. D 73 (2006) 015001 [hep-ph/0509118] [INSPIRE]. 